\title{
RESEARCH IN NUCLEAR PHYSICS
}

\section{FINAL REPOR T}

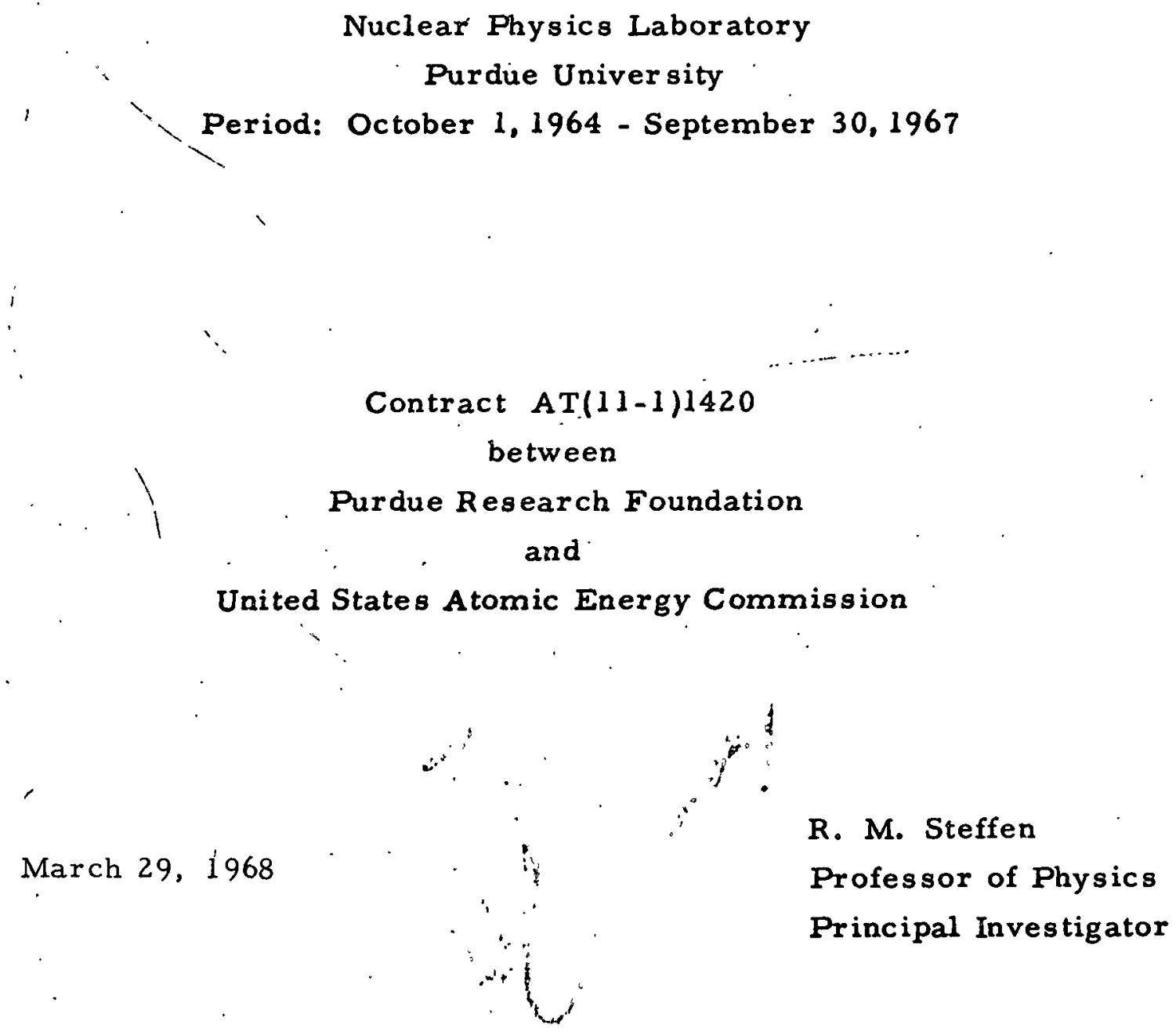




\section{DISCLAIMER}

This report was prepared as an account of work sponsored by an agency of the United States Government. Neither the United States Government nor any agency Thereof, nor any of their employees, makes any warranty, express or implied, or assumes any legal liability or responsibility for the accuracy, completeness, or usefulness of any information, apparatus, product, or process disclosed, or represents that its use would not infringe privately owned rights. Reference herein to any specific commercial product, process, or service by trade name, trademark, manufacturer, or otherwise does not necessarily constitute or imply its endorsement, recommendation, or favoring by the United States Government or any agency thereof. The views and opinions of authors expressed herein do not necessarily state or reflect those of the United States Government or any agency thereof. 


\section{DISCLAIMER}

Portions of this document may be illegible in electronic image products. Images are produced from the best available original document. 


\section{PREFACE}

This report reviews the research and technical development conducted at the Nuclear Physics Laboratory of Purdue University during the period from October 1, 1964 to September 30,1967. Two accelerators were used in this research program, the 37-inch Purdue Cyclotron and the ENTandem Van-deGraaff of the Argonne National Laboratory. The installation of the Purdue FN-Tandem Accelerator, with funds provided by the National Science Foundation, is in progress, and it is expected to be available for research in early 1969.

Most of the research reviewed in this report has been reported in the annual Progress Reports No. 15 (June 1965), No. 16 (June 1966) and No. 17 (June 1967).

Research at the Nuclear Physics Laboratory is performed by the faculty and graduate students of the Department of Physics of Purdue University. Support is provided by the State of Indiana and the U.|S. Atomic Energy Commission.

The investigations described in this report partly continue and extend the experimental and theoretical work described in earlier Progress Reports (No. 1 to 14) under contracts $A T(11-1) 123$, Task $B$ and $A T(11-1) 122$. Continued emphasis is placed on elastic and inelastic scattering of $\alpha$-particles and deuterons, pick-up and stripping reactions, angular correlation measurements involving beta particles, conversion electrons, gamma radiation, and studies of perturbations by extra nuclear fields.: A new project has been started on Coulomb Excitation by heavy ions. An extensive program in eleo-

tronics instrumentation using the latest developments of the art has been initiated and is described under instrumentation.

This report concludes with a list of the Purdue Nuclear Physics Laboratory Personnel, a list of advanced degrees granted during the period of the report, and a list of publications. 
I. NUCLEAR REACTION STUDIES (CYCLOTRON) • • . . . . . . . 1

A. Isospin Conservation in the $\mathrm{Ca}^{40}(\mathrm{~d}, \alpha) \mathrm{K}^{38}$ Reaction . . . . .. . 1

B. $\mathrm{F}^{19}(\mathrm{~d}, \alpha) \mathrm{O}^{17}$ Reaction at $9.2 \mathrm{MeV}$. . . ... . . . . . 2

C. Experimental Study of the $2 I+1$ Rule Using the $(d, \alpha)$ Reaction on $\mathrm{F}^{19}, \mathrm{Na}^{23}, \mathrm{Al}^{27}$, and $\mathrm{P}^{31} \cdot . \cdot . \cdot . \cdot .3$

D. Some $(d, \alpha)$ Differential Cross Sections for $\mathrm{Na}^{23}, \mathrm{Al}^{27}$, and $\mathrm{P}^{31}$ at About $9.3 \mathrm{MeV}$. . . .......... 4

E. Optical-Model and Distorted-Wave Analyses of Some

18-MeV Alpha-Particle Scattering Cross Sections for $\operatorname{Ar}^{40}, \mathrm{~S}^{32}, \mathrm{P}^{31}, \mathrm{Na}^{23}$, and $\mathrm{Ne}^{20} \cdot . . . . . .5$

F. Elastic and Inelastic Scattering of 9.8-MeV Deuterons from $P^{31}$. . . . . . . . . . . . . . . 6

G. Study of the $\mathrm{P}^{31}\left(\alpha, \mathrm{d}_{0,1}\right) \mathrm{S}^{33}$ Reactions at $18.7 \mathrm{MeV} \cdot . \cdot . \quad .7$

H. Measurement and Distorted-Wave Born-Approximation

Analyses of the 18.7-MeV Cross Sections for the $\mathrm{Na}^{23}\left(\alpha, \mathrm{d}_{0,1}\right) \mathrm{Mg}^{25}$ Reactions . . . . . . $\cdot{ }^{2}, 8$

I. Measurements and DWBA Analyses of Some 13.9-MeV Differential Cross Sections for the $\mathrm{N}^{14}\left(\mathrm{He}^{3}, \alpha\right) \mathrm{N}^{13}$

Reactions

J. Study of Some $\mathrm{C}^{12}\left(\mathrm{He}^{3}, \alpha\right) \mathrm{C}^{11}$ Reactions at $13.9 \mathrm{MeV}$. . . . . .11

K. The $\mathrm{K}^{39}\left(\alpha, \alpha^{\prime}\right) \mathrm{K}^{39}$ and $\mathrm{K}^{39}(\alpha, \mathrm{p}) \mathrm{Ca}^{42}$ Reactions . . . . . . 12

L. Isotopic Spin Conservation in the $K^{39}(d, \alpha) A^{37}$ Reaction . . . . 13

M. An Analysis of the Elastic and Inelastic Scattering . of Alpha Particles by 'F' Nuclei . . . . . . . . . . . 14 
II. COULOMB EXCITATION STUDIES • . • • • • • • • • • . . . 17

A. Static Quadrupole Moment of the $2^{+}$State in Cd 14

Determined by Coulomb Excitation . . . . . . . . 17

B. Static Quadrupole Moment of the First Excited

$$
2^{+} \text {State in } \mathrm{Fe}^{56} \text {. . . . . . . . . . . . . . }
$$

III. ANGULAR CORRELATION STUDIES . . . . . . . • . . . • . 24

A. Search for Higher Order Effects in Allowed 8 Transitions . - . 24

B. Higher Order Effects in $\beta-\gamma$ Correlations Involving

Allowed $\beta$ Transitions . . . . . . . . . . . 25

C. Study of Second Excited $2^{+}$States of Some Even-Even

Nuclei by Beta-Gamma Angular Correlations . . . . . 26

D. Nuclear Matrix Elements in the First-Forbidden

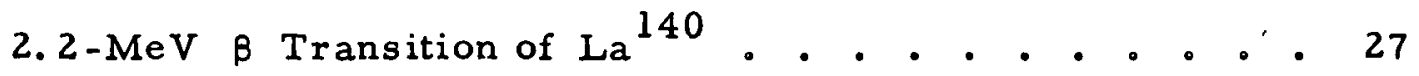

E. Beta-Gamma Circular Polarization Correlation of $\mathrm{Sb}^{122} \ldots \ddots^{\prime}{ }^{-} 28$

F. Energy Dependence of Beta-Gamma Circular Polarization 29

G. Transverse Polarization of $\mathrm{K}$-Conversion Electrons

Following the $\beta$ Decay of $\mathrm{Au}^{198}$. . . . . . . . . 31

H. Conversion-Electron Particle Parameters in the

Decay of $\mathrm{Eu}^{152}$ and $\mathrm{Eu}^{154}$. . . . . . . . . . 32

I. Conversion-Electron Gamma Directional Correlations in $\operatorname{Tm}^{169}$ and $\mathrm{Lu}^{175}$. . . . . . . . . . . . 33

J. $K L$ and $M$ Internal Conversion Parameters of M4 Transitions . . . . . . . . . . . . . . 35

K. Beta-Gamma Directional Correlations in the Decay of Sb ${ }^{124}$. . 37

L. Beta-Gamma Directional Correlation of K-Forbidden

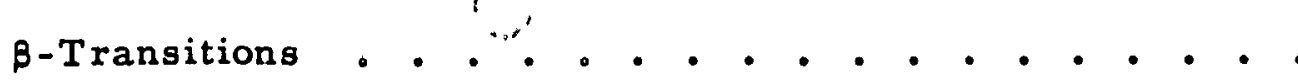


M. Gamma-Gamma Directional Correlation Measurements with a $\mathrm{Ge}(\mathrm{Li})-\mathrm{NaI}(\mathrm{T} \ell)$ Correlation Equipment . . . . . 40

N. Gamma-Gamma Directional Correlation Measurements with a $\mathrm{Ge}(\mathrm{Li})-\mathrm{Ge}(\mathrm{Li})$ Corellation Equipment . . . . . . 41

O. Mixing Ratio of $\operatorname{Transitions}$ in the Decay of $\mathrm{Ir}^{192}$. . . . . . 42

P. The g-Factors of $2^{+}$Vibrational States in $\mathrm{Pt}^{192}$. . . . . . . 44

Q. The Magnetic Hyperfine Field at Hf Nuclei in an Iron Lattice . . . . . . . . . . . . . . . 47

R. Alpha-Gamma Directional Correlation Measurement in the Decay of $\mathrm{Th}^{230}$

S. Hyperfine Field of $\mathrm{Rh}^{103}$ in Ir on and the $\mathrm{Rh}^{103}-\mathrm{Rh}^{100 \mathrm{~m}}$

Hyperfine Anomaly in Iron . . . . . . . . . . . . 48

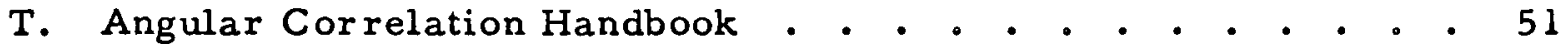

IV. GENERAL NUCLEAR SPECTROSCOPY . • . • • • • • • . . 52

A. Short Lifetime Measurements . . . . . . . . . . 52

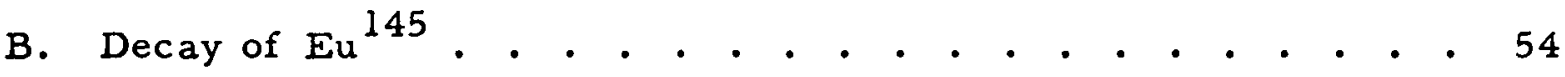

V. INSTRUMENTATION • • • • • • • • • • • • • • • • •

A. The Purdue Broad-Range Magnetic Spectrograph . . . . . 55

B. Construction of 18 " Scattering Chamber . . . . . . . . . 57

C. Electronic Instrument Development • • • • • • • • • • • 58

D. Fabrication of Lithium-Drifted Germanium Detectors . . . . 62

VI. COMPUTER PROGRAMS FOR DATA ANAL.YSIS • • • • • • • • 63

A. Non-Relativistic Kinematics for Nuclear Reactions -

Two-Body Final States . . . . . . . . . . . 63 
B. Non-Relativistic Reaction Kinematics for Nuclear Reactions Three-Body Final States . . . . . . . . . . 63

C. Finite Target Thickness Calculation . . . . . . . . : 64

D. Program for Analysis of Angular Correlation Data . . . . 65

E. Program for Analysis of $\beta-\gamma$ Angular Correlation Data

in Terms of $\beta$-Matrix Elements . . . . . . . . 66

F. Program for Computation of Internal Conversion Coefficients

and Directional Correlation Particle Parameters

Including Higher Order Effects . . . . . . . . . 67

VII. THEORY • . . . . . . . . . . . . . . . . . . 68

A. Large-Spin Rotational States of Deformed Nuclei. - . . . 68

B. Higher-Order Corrections to the Cranking Model. . . . . 69.

C. Quantum-Mechanical Model of Mössbauer Line Narrowing . . 70

D. Perturbation of Angular Correlations by Rhombic

Electric Field Gradients . . . . . . . . . . 70

E. Theory of Angular Distributions and Correlations of

Nuclear Radiations . . . . . . . . . . . 72

F. Calculations of Internal Conversion Coefficients and

Particle Parameters for Finite Size Nuclei. . . . . . 72

VIII. PERSONNEL OF PURDUE NUCLEAR PHYSICS LABORATORY • . 76

A. Faculty. . . . . . . . . . . . . . . . 76

B. Post-Doctoral Research Associates .. • . . . . . . . 76

C. Graduate Research Assistants.'. . . . . . . . . . 76

D. Cyclotron Staff. • • • • • • • • • • • • • • •

E. Electronics Shop . . . . . . . . . . . . . . . 78 
F. Plate Readers . . . . . . . . . . . . . . 78

G. Secretarial • . . . . . . . . . . . . . . 78

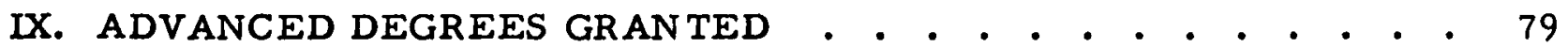

X. LIST OF PUBLICATIONS . . . . . . . . . . . . . . . . . . 80

A. Papers Published in Professional Journals . . . . . . . . 80

B. Reports and Tables . . . . . . . . . . . . . . 83
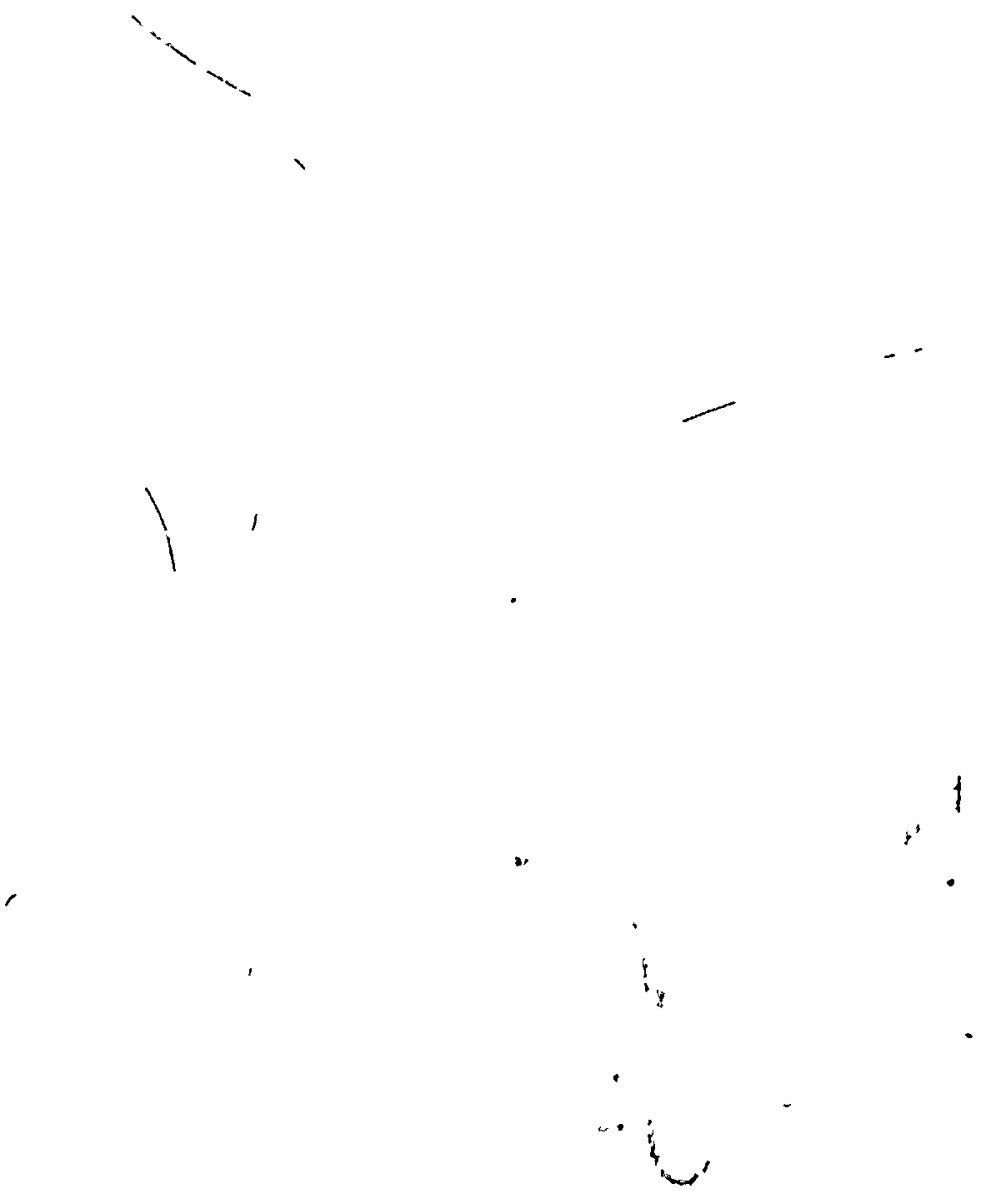
A. Isospin Conservation in the $\mathrm{Ca}^{40}(\mathrm{~d}, \alpha) \mathrm{K}^{38}$ Reaction

J. W. Hemsky, E. Bleuler ${ }^{*}$ and D. J. Tendam

The angular distributions of the five most energetic alpha groups emitted in the reaction $\mathrm{Ca}^{40}(\mathrm{~d}, \alpha) \mathrm{K}^{38}$ at an incident energy of $9.20 \mathrm{MeV}$ were measured with a broad-range spectrograph from $15^{\circ}$ to $155^{\circ}$. The $\Delta T=0$ is ospin selection rule is found to inhibit the transition to the $\mathrm{J}^{\pi}, \mathrm{T}=0^{+}$, 1 state at $0.129 \mathrm{MeV}$ by about a factor 3. The corresponding isospin mixing in the compound nucleus, $20-25 \%$, is considerably smaller than the nearly complete mixing previously reported by Janecke for a deuteron energy of 7. $7 \mathrm{MeV}$. This could be the predicted decrease at high excitation energies in the compound nucleus where $\left\langle\mathrm{H}_{\mathrm{c}}\right\rangle\langle\langle\Gamma\rangle$. The angular distribution is nearly symmetric about $90^{\circ}$, but a fit with a quadratic form of associated Legendre polynomials $\mathrm{P}_{\mathrm{J}}^{\mathrm{l}}(\cos \theta)$ required very large cross terms which may indicate a deviation from a statistical compound-nucleus reaction larger than expected from the limited energy spread (about two coherence widths).) The angular distribution of the transition to the $2^{+}$, l state at $2.406 \mathrm{MeV}$ has forward peaking and may have a direct interaction component in approximate agreement with the impurities in the initial and final states. A comparison of the angular distributions for the transitions to the three lowest $T=0$ states at $E_{\mathrm{d}}=9.20 \mathrm{MeV}$ with previous work at $7.7,11.4$ and $14.8 \mathrm{MeV}$ does not show any obvious general trends.

The results of this investigation have been published in Nuclear Physics, Al05, 605 (1967)

* Present address, Pennsylvania State University, State College, Pennsylvania. 


\section{B. $\quad F^{19}(d, \alpha) O^{17}$ Reaction at $9.2 \mathrm{MeV}$}

S. W. Cosper, B. T. Lucas, and O. E. Johnson

The differential cross sections for the $F^{19}(d, \alpha) O^{17}$ reaction which leave $\mathrm{O}^{17}$ in its ground and lowest four excited states have been measured using silicon surface-barrier detectors. Thin Teflon targets $(370$ to 720 $\mu \mathrm{g} / \mathrm{cm}^{2}$ ) were bombarded with 9. $2 \mathrm{-MeV}$ deuterons and alpha spectra were obtained at 46 laboratory angles between 10 and $172.5^{\circ}$. The five angular distributions exhibited forward and backward peaking, an over-all oscillatory structure, and minima whose magnitudes differ significantly from zero. The angular distributions are analyzed in terms of an expression which is the sum of an isotropic term and one which arises from the simultaneous action of twonucleon pickup and heavy-particle stripping (HPS) mechanisms. Good fits are obtained for the $\alpha_{0}, \alpha_{2}$, and $\alpha_{4}$ distributions, but only the gross features of the $\alpha_{1}$ and $\alpha_{3}$ distributions could be fitted. A discussion regarding the identification of either the pickup or the knockout mechanism as the dominant forward-angle direct-interaction process for $(d, \alpha)$ reactions in light nuclei is presented. It is concluded that the experimental angular distributions are fitted equally well irrespective of which one is assumed to act along with HPS. An interpretation of the differential cross sections is made which is based on the speculation that direct-interaction and statistical compound-nucleus (SCN) processes contribute incoherently. A method for the decomposition of the cross section is proposed in which the SCN contribution is assumed to be isotropic. The integrated SCN component of the cross sections is shown to be closely proportional to $2 I+1$.

The results of this investigation have been published in The Physical Review, 138, B51, (1965). 


\section{Experimental Study of the $2 I+1$ Rule Using the $(\mathrm{d}, \alpha)$ Reaction on $\mathrm{F}^{19}, \mathrm{Na}^{23}, \mathrm{Al}^{27}$, and $\mathrm{P}^{31}$}

S. W. Cosper and O. E. Johnson

The angular distributions corresponding to the $(d, \alpha)$ production cross sections $\left(E_{d} \approx 9.3 \mathrm{MeV}\right)$ for the ground states and some of the low lying states of $\mathrm{O}^{17}, \mathrm{Ne}^{21}, \mathrm{Mg}^{25}$, and $\mathrm{Si}^{29}$ have been measured using silicon surface-barrier detectors. The discussion of the $2 I+1$ rule within the context of these experimental observations is based on the premise that both the direct interaction (DI) and compound nucleus (CN) mechanisms contribute incoherently to the angular distributions, and the approximate validity of an ad hoc method used for the decomposition of these differential cross sections into $\mathrm{DI}$ and $\mathrm{CN}$ components. The integrated experimental differential cross sections, $\sigma_{T}\left(10^{\circ}-170^{\circ}\right)$ and $\sigma_{B}\left(90^{\circ}-170^{\circ}\right)$, and the integrated $C N$ component, $\sigma_{C N}\left(10^{\circ}-170^{\circ}\right)$, are analyzed in terms of the 2 I 1 rule: The $\sigma_{\mathrm{CN}}$ values are found to be more nearly proportional to $2 I+1$ than either the $\sigma_{T}$ or $\sigma_{B}$ values. This result is interpreted as indicating that the $2 I+1$ dependence of the $(d, \alpha)$ cross sections is a characteristic associated with the CN mechanism rather than the DI mechanism. Furthermore, it is concluded that spin assignments based solely on the assumption of a 2 It 1 dependence of either the $\sigma_{T}$ or $\sigma_{B}$ values derived from $(\alpha, \alpha)$ reactions would not as a rule be very reliable.

The results of this investigation have been published in The Physical Review, 138, B 610, (1965). 
D. Some $(\alpha, \alpha)$ Differential Cross Sections for $\mathrm{Na}^{23}, \mathrm{Al}^{27}$, and $\mathrm{P}^{31}$ at About $9.3 \mathrm{MeV}$

S. W. Cosper, B. T. Lucas, and O. E. Johnson

The differential cross sections for the $(d, \alpha)$ reactions which lead to various nuclear states in $\mathrm{Ne}^{2 \mathrm{l}}$ (ground, 1, and 2), $\mathrm{Mg}^{25}$ (ground, 1, 2, 3, and 4), and $\mathrm{Si}^{29}$ (ground, $1,2,3,4,5$, and 6) were measured at nominal deuter on energies of $9.2,9.2$, and $9.5 \mathrm{MeV}$, respectively, using a silicon surface-barrier detector. Targets of various thicknesses ranging from 70 to $642 \mu \mathrm{g} / \mathrm{cm}^{2}$ were used. In each case the differential cross section was determined at more than 34 angles from 10 to $172.5^{\circ}$. Although the shape of each angular distribution is different from any of the others in detail, all exhibit the following characteristics: pronounced forward and backward peaking; an over-all oscillatory structure; an asymmetry about $90^{\circ}$; and minima whose magnitudes differ significantly from zero. Comparisons are madewith $(d, \alpha)$ results which have been previously obtained by other investigators using deuteron energies in the 7-15-MeV range. No correlation could be established between any specific detail in shape or general feature of these angular distributions and any physical quantity associated with the nuclear states involved. Analyses of these differential cross sections are described using an expression consisting of the sum of an isotropic component and a plane-wave Born approximation component which arises from the coherent action of a two-nucleon pickup and a heavy-particle-stripping mechanism.

The results of this investigation have been published in The Physical Review, 139. B763, (1965). 
E. Optical-Model and Distorted-Wave Analyses of Some $18-\mathrm{MeV}$

Alpha-Particle Scattering Cross Sections for

$\mathrm{Ar}^{40}, \mathrm{~S}^{32}, \mathrm{P}^{31}, \mathrm{Na}^{23}$, and $\mathrm{Ne}^{20}$

B. T. Lucas, S. W. Cosper, and O. E. Johnson

The 18-MeV elastic and inelastic alpha-particle cross sections for some states of $\mathrm{Ne}^{20}$ (Gnd., 1.632 MeV), $\mathrm{Na}^{23}$ (Gnd., 0.439, 2. $080 \mathrm{MeV}$ ), $\mathrm{P}^{31}$ (Gnd., 1.265, 2. $232 \mathrm{MeV}$ ), $\mathrm{S}^{32}$ (Gnd.), and $\operatorname{Ar}^{40}$ (Gnd., 1.462 MeV) have been analyzed in terms of the nuclear optical model and the distortedwave theory. Although good qualitative agreement between the empirical and optical-model cross sections is found, particularly at forward angles, 'the quantitative agreement is significantly poorer than that found for hea vier target nuclei at higher incident energies. Further intercomparison of the results of the present and previous studies strongly suggests that for the prevailing experimental and physical circumstances $\left(E_{\alpha} \approx 18 \mathrm{MeV}\right.$ and $A<40$ ) of the present investigation the applicability of the optical model is marginal. The distorted-wave predictions are in fair agreement with the experimental data at the more forward angles $\left(\theta \lesssim 80^{\circ}\right)$ in that they generally reproduce the positions and relative amplitudes of two or more of the maxima. For larger angles $\left(\theta>80^{\circ}\right)$ the quality of the fits deteriorates insofar as detall is concerned; however, the calculated curves do follow the general trend of the data. For the inelastic angular distributions the quality of the fits is poorer and the angular range over which the fits are acceptable is smaller than for the corresponding elastic cross sections.

The results of this investigation have been published in The Physical Review, 144, 972, (1966). 
F. Elastic and Inelastic Scattering of $9.8-\mathrm{MeV}$ Deuterons from $\mathrm{P}^{31}$
B. T. Lucas and O. E. Johnson

The 9.8-MeV differential cross sections for deuteron scattering reactions which leave $\mathrm{P}^{31}$ in its ground and lowest three excited states (1. 265, 2.232, and $3.133 \mathrm{MeV}$ ) have been measured. The targets were produced by the vacuum evaporation of red phosphorous $\left(\sim 625 \mu \mathrm{g} / \mathrm{cm}^{2}\right)$ onto thin Formvar films $\left(2-5 \mu \mathrm{g} / \mathrm{cm}^{2}\right)$. The spectra were measured using an electronic mass-discriminating spectrometer system which incorporated an $(E, \Delta E)$ counter telescope consisting of two silicon surface-barrieridetectors. The $\left(\sigma_{E} / \sigma_{R}\right)$ ratio for the elastic scattering exhibits a somewhat " irregular oscillatory structure forward of $100^{\circ}$. The $d_{1}$ and $d_{2}$ angular distributions are remarkably similar in shape and are clearly in phase, but there is no definite phase relationship between them and the $d_{0}$ angular distribution. The $\mathrm{d}_{3}$ angular distribution is roughly out of phase with the $d_{1}$ and $d_{2}$ angular distributions. The integrated cross sections for the excitation of the first, second, and third excited states are $3.51 \pm 0.04 \mathrm{mb}$ $\left(26.7^{\circ}-170.7^{\circ}\right), 6.74 \pm 0.04 \mathrm{mb}\left(26.8^{\circ}-170.7^{\circ}\right)$, and $0.77 \pm 0.02 \mathrm{mb}\left(32.3^{\circ}\right.$ $170.8^{\circ}$ ), respectively. The elastic-scattering cross section was analyzed in terms of the nuclear optical model with a four-parameter Saxon potential. Three distinct sets of parameters were found which give moderately good fits to the experimental data for angles less than $150^{\circ}$. The ambiguities in the optical-model parameters encountered in the fitting procedure are described and discussed. The $d_{1}$ and $d_{2}$ cross sections were analyzed in terms of the distorted-wave theory using a simple collective-model description for the excited states of $\mathrm{P}^{31}$ and the optical-model parameters determined 
through the analysis of the elastic-scattering data. The resulting calculated cross sections reproduce the general features of the data over a rather wide angular range, the most serious discrepancies occurring at forward angles. The implications of these analyses and the present experimental results are discussed in terms of existing collective-model descriptions of this nucleus.

The results of this investigation have been published in The Physical Review, $145,887,(1966)$.

G. Study of the $\mathrm{P}^{31}\left(\alpha, \mathrm{d}_{0,1}\right) \mathrm{S}^{33}$ Reactions at $18.7 \mathrm{MeV}$

B. B. Srivastava, S. W. Cosper, and O. E. Johnson

The 18. $72-\mathrm{MeV}$ differential cross sections for the $\mathrm{P}^{31}(\alpha, \mathrm{d}) \mathrm{S}^{33}$ reactions leading to the ground and $0.841-\mathrm{MeV}$ states of $\mathrm{S}^{33}$ have been measured from $15^{\circ}$ to $170^{\circ}$ at $5^{\circ}$ intervals. The deuteron spectra were determined using a spectrometer configuration which incorporated an $E \times \Delta E$ mass identification system and an $(E, \Delta E)$ counter telescope consisting of two silicon surface-barrier detectors. The target was prepared by the thermal vacuum evaporation of red phosphorus $\left(650 \pm 12 \mu \mathrm{g} / \mathrm{cm}^{2}\right)$ onto a thin Formvar film $\left(\sim 20 \mu \mathrm{g} / \mathrm{cm}^{2}\right)$. In contrast to the well-defined oscillatory shape of the angular distribution associated with the transition to the $0.841-\mathrm{MeV}$ state, that corresponding to the transition to the ground state has only washed-out undulations. The integrated cross sections for the ground and $0.841-\mathrm{MeV}$ state reactions are $724 \pm 16 \mu \mathrm{b}\left(16.9^{\circ}-171.3^{\circ}\right)$ and $425 \pm 10 \mu \mathrm{b}\left(17.0^{\circ}-171.3^{\circ}\right)$, respectively. An analysis of the data in the distorted-wave Born approximation has been made in terms of a zero-range, 
knock-out model in which the initial (final) nuclear state is' represented as a two-body system with a deuteron (alpha particle) bound to a $\mathrm{Si}^{29}$ core. Good agreement between the experimental and theoretical angular distributions was achieved. The analyses require a $2 \mathrm{~s}_{1 / 2}$ state for the $\left(\mathrm{d}, \mathrm{Si}^{29}\right)$ system representing the ground state of $\mathrm{P}^{31}$, and $l_{3 / 2}$ and $2 \mathrm{~s}_{1 / 2}$ states for the $\left(\alpha, \mathrm{Si}^{29}\right)$ system representing the ground and $0.84 \mathrm{l}-\mathrm{MeV}$ states of $\mathrm{s}^{33}$, respectively. An interpretation of the experimental results on the basis of the dominant shell-model configurations for the states of $\mathrm{P}^{31}$ and $\mathrm{S}^{33}$ yields the conclusion that there is no enhancement of the transition in which the members of the transferred nucleon pair enter equivalent orbitals relative to that in which the nucleons enter nonequivalent orbitals.

The results of this investigation have been published in The Physical Review, $153,1221,(1967)$.

H. Measurement and Distorted-Wave Born-Approximation Analyses of the 18. 7-MeV Cross Sections for the $\mathrm{Na}^{23}\left(\alpha, \mathrm{d}_{0,1}\right) \mathrm{Mg}^{25}$ Reactions

B. B. Srivastava and O. E. Johnson

The 18. 7-MeV differential cross sections for the $\mathrm{Na}^{23}\left(\alpha, \mathrm{d}_{0,1}\right) \mathrm{Mg}^{25}$ reactions were determined at $5^{\circ}$ intervals over the angular range from about $25^{\circ}$ to $170^{\circ}$. The spectrometer system included an $(\mathrm{E} \times \Delta \mathrm{E})$ particle identifier, an $(E, \Delta E)$ counter telescope consisting of two silicon surface-barrier detectors, and a multichannel pulse-height analyzer. One target each of sodium hydride $\left(266 \pm 10 \mu \mathrm{g} / \mathrm{cm}^{2}\right)$ was used. The target material was vaporized in a vacuum and condensed onto a thin $\left(\sim 20 \mu \mathrm{g} / \mathrm{cm}^{2}\right)$ Formvar substrate. The integrated 
cross sections for the transitions to the ground and $0.584-\mathrm{MeV}$ states of $\mathrm{Mg}^{25}$ are $1337 \pm 30 \mu \mathrm{b}\left(23.1^{\circ}\right.$ to $\left.171.6^{\circ}\right)$ and $349 \pm 9 \mu \mathrm{b}\left(34.7^{\circ}\right.$ to $\left.171.6^{\circ}\right)$ respec tively. The angular distributions are similar in over-all shape and exhibit a somewhat washed-out structure with strong backward-angle peaking. This similarity could not be correlated in a simple way with any of the known properties of the nuclear states. Distorted-wave Born-approximation analyses of the data have been made in terms of both zero-range knockout and zero-range stripping models. In the knockout model, the initial (final) nuclear state is described as a two-body system consisting of a deyteron (an $\alpha$ particle) bound to a $\mathrm{Ne}^{21}$ core, and in the stripping model the final nuclear state is described as a deuteron bound to a $\mathrm{Na}^{23}$ core. Using reasonable parameter values, the gross features of both angular distributions could be marginally reproduced by the knockout model but not by the stripping model. The knockout analyses require a $\lg _{3 / 2}$ state for the $\left(d, \mathrm{Ne}^{21}\right)$ system representing the ground state of $\mathrm{Na}^{23}$, and require $2 \mathrm{~d}_{5 / 2}$ and $2 \mathrm{~d}_{1 / 2}$ states for the $\left(\alpha, \mathrm{Ne}^{2 \mathrm{l}}\right)$. system representing the ground and $0.584-\mathrm{MeV}$ states of $\mathrm{Mg}^{25}$ respectively. The results of this investigation have been published in The Physical Review, 156, $1219,(1967)$.

I. Measurements and DWBA Analyses of Some 13.9-MeV Differential Cross Sections for the $\mathrm{N}^{14}\left(\mathrm{He}^{3}, \alpha\right) \mathrm{N}^{13}$ Reactions

B. T. Lucas, D. R. Ober, and O. E. Johnson

The $\left(\mathrm{He}^{3}, \alpha\right)$ differential cross sections leading to the Gnd., 2.367-, $(3.51+3.56)-, 6.38-$, and $(7.18+7.42)-\mathrm{MeV}$ states of $\mathrm{N}^{13}$ have been measured at an incident $\mathrm{He}^{3}$ energy of $13.9 \mathrm{MeV}$ using silicon surface-barrier 
detectors and a conventional electronic spectrometer system. Energy spectra were accumulated at $2.5^{\circ}$ intervals over a laboratory angular range from $17.5^{\circ}$ to $90^{\circ}$ and at $5^{\circ}$ intervals from $90^{\circ}$ to $170^{\circ}$. The experimental differential $\left(\mathrm{He}^{3}, \alpha\right)$ cross sections corresponding to states in $\mathrm{N}^{13}$ at $0,2.367,6.38$, and $(7.18+7.42) \mathrm{MeV}$ exhibit a pronounced oscil latory structure, suggesting that a direct-reaction mechanism is dominant. The composite cross section corresponding to the transitions leading to the $(3.51+3.56)-\mathrm{MeV}$ doublet has a somewhat washed-out structure. All angular distributions display a definite forward-angle peaking, and those leading to the Gnd., 2.367-, and $(3.51+3.56)-\mathrm{MeV}$ states of $\mathrm{N}^{13}$ show evidence of strong backward-angle peaking. The transitions leading to the odd-parity states in $\mathrm{N}^{13}$ appear to be considerably enhanced relative to those leading to the even-parity states. The angular distributions corresponding to the transitions to the Gnd.,2.367-, and 6.38-MeV states have been analyzed within the framework of the aero-range distorted-wave theory using a simple knockout model. The use of cutoff radii equal to or slightly larger than the nuclear radius was necessary in order to obtain reasonably good representations of the experimental data.

The results of this investigation have been submitted to The Physical Review for publication. 
J. Study of Some $\mathrm{C}^{12}\left(\mathrm{He}^{3}, \alpha\right) \mathrm{C}^{11}$ Reactions at $13.9 \mathrm{MeV}$

D. R. Ober and O. E. Johnson

The 13. 9-MeV angular distributions for the $\mathrm{C}^{12}\left(\mathrm{He}^{3}, \alpha\right) \mathrm{C}^{11}$. reactions which produce the ground, 2.00-, 4.32-, and 4.81-MeV states of $\mathrm{C}^{11}$ were measured using silicon surface-barrier detectors. The differential cross sections were determined at $2.5^{\circ}$ intervals over a laboratory angular range from about $15^{\circ}$ to $100^{\circ}$. The angular distributions display features which suggest that a direct-reaction mechanism is operative. The ground-state angular distribution is strongly peaked at forward angles with a weak washed-out oscillatory structure, and those for the excited states are also forward peaked, but have a strong well-defined oscillatory nature. The integrated cross sections over the common center of-mass angular range $\left(32^{\circ}\right.$ to $\left.112^{\circ}\right)$ for the ground and first three excited states are $30.5,9.2,9.7$, and $11.9 \mathrm{mb}$, respectively. Each angular dis tribution was analyzed in terms of both zero-range distorted-wave Bornapproximation knockout and pickup models. Reasonable correspondence between theory and experiment for the ground-state $\left(3 / 2^{-}\right)$and secondexcited-state $\left(5 / 2^{-}\right)$angular distributions was achieved only with the pickup model, while only the knockout model provided a reasonable representation of the angular distributions corresponding to the first $\left(1 / 2^{-}\right)$and third $\left(3 / 2^{-}\right)$ excited states.

The results of this investigation have been submitted to The Physical Review for publication. 
K. The $\mathrm{K}^{39}\left(\alpha, \alpha^{\prime}\right) \mathrm{K}^{39}$ and $\mathrm{K}^{39}(\alpha, \mathrm{p}) \mathrm{Ca}^{42}$ Reactions

\author{
G. F. Dell, Jr.
}

Through the use of the Purdue broad-range magnetic spectrograph, the reactions $\mathrm{K}^{39}\left(\alpha, \alpha^{\prime}\right) \mathrm{K}^{39}$ and $\mathrm{K}^{39}(\alpha, \mathrm{p}) \mathrm{Ca}^{42}$ have been investigated. The targets consisted of high-purity metallic potassium vacuum deposited on a thin Formvar substrate. To facilitate the separation of the desired peaks from those resulting from target contaminants, the spectra of reaction particles were measured at scattering angles of $53^{\circ}, 57^{\circ}$, and $60^{\circ}$; in order to eliminate the spectral peaks arising from the alpha-particle and deuteron exit channels, two spectra were recorded with a 5-mil Mylar foil in front of the nuclear-track emulsions. The spectra were observed over a momentum range corresponding to excitation energies between 2.5 and 10. $5 \mathrm{MeV}$ in the residual nucleus.

The target thickness was determined by the simultaneous solution of the kinematics equation and the energy-loss equations for the reaction particles; the experimentally determined values of the elastic-scattering cross sections for alpha particles on $\mathrm{C}^{12}, \mathrm{O}^{16}$, and $\mathrm{K}^{39}$ were used in this procedure.

The spectrum analysis has been accomplished primarily by means of a kinematics program which includes corrections for energy losses in the target. Peaks which correspond to known levels in the principle target contaminants were eliminated and were not subjected to further analysis. The principle target contaminants are $\mathrm{C}^{12}, \mathrm{~N}^{14}$, and $\mathrm{O}^{16}$; however, the $\mathrm{O}^{16}(\alpha, p) \mathrm{F}^{19}$ reaction has no open channels in the momentum range under consideration. 
To date, nineteen levels between 4.04 and $\left.9.5 \mathrm{MeV}\right|_{\text {in }}$ the $\mathrm{Ca} 42$ nucleus have been identified and two levels between 4.12 and $4.8 \mathrm{MeV}$ in the $\mathrm{K}^{39}$ nucleus have been observed. Hopefully, more levels in the $\mathrm{K}^{39}$ nucleus will be identified, but due to the presence of strong peaks arising from the low-lying levels in $\mathrm{C}^{12}$ and $\mathrm{O}^{16}$, the number of identifiable levels in $\mathrm{K}^{39}$ will be limited.

I. Is otopic Spin Conservation in the $K^{39}(d, \alpha) A^{37}$ Reaction

\section{L. Wiley}

The lowest $T=3 / 2$ state in $A^{37}$ should appear at an excitation energy of approximately $5 \mathrm{MeV}$, and preliminary measurements indicate that the reaction cross sections in this energy region would be sufficiently high to permit a detailed investigation of the level scheme in $\mathrm{A}^{37}$.

After the preliminary data had been collected for an incident deuteron energy of $9.5 \mathrm{MeV}$, an abstract of a paper describing the $\mathrm{K}^{39}(\mathrm{~d}, \alpha)$ $A^{37}$ reaction for $E_{d}=10 \mathrm{MeV}$ appeared in the Bulletin of the American Physical Society. 1) The experimental details and the analysis of the data from this work at the California Institute of Technology were so similar to those of the experiment under way here that it was decided to abandon this investigation at Purdue.

1. J. H. McNally, Bull. Am. Phys. Soc. 9, 553, (1964). 
M. An Analysis of the Elastic and Inelastic Scattering of Alpha Particles by $F^{19}$ Nuclei

\section{L. Wiley}

The spin, parity, and excitation-energy assignments, denoted by $\left(J^{\pi}, E\right)$, of the six lowest-lying levels in the $F^{19}$ nucleus have been reported to be the following: ground state $\left(1 / 2^{+}, 0.0000 \mathrm{MeV}\right)$, first excited state $\left(1 / 2^{-}, 0.10987 \mathrm{MeV}\right)$, second excited state $\left(5 / 2^{+}, 0.1970 \mathrm{MeV}\right)$, third excited state $\left(5 / 2^{-}, 1.3466 \mathrm{MeV}\right)$, fourth excited state $\left(3 / 2^{-}, 1.4601 \mathrm{MeV}\right)$, and fifth excited state $\left(3 / 2^{+}, 1.5559 \mathrm{MeV}\right) .{ }^{1)}$ These six levels fall into two natural groupings, each group (a triplet) containing three levels spaced approximately $100 \mathrm{keV}$ apart.

In order to resolve the peaks corresponding to the alpha-particle groups arising from reactions leading to the various low-lying levels in the $F^{19}$ nucleus, it was necessary to use the Purdue broad-range magnetic spectrograph for this investigation. To minimize the impairment of the resolution due to a spread in the energy distribution as the incident and reaction particles traversed the target, it was necessary to obtain fluorinated targets which were as thin as was practical--considering the reaction cross sections, the incident-beam current, and the beam time available for this experiment. At the time this experiment was being planned, 1/4-mil Teflon was the thinnest fluorinated-hydrocarbon foil available commercially. Since this foil is much too thick to serve as a target for this experiment, it was necessary to develop a technique for fabricating fluorinated targets whose thickness was of the order of $75 \mu \mathrm{gm} / \mathrm{cm}^{2}$. The final targets consisted of thin, multi-layered, Kynar-plastic foils produced in this laboratory. ${ }^{2}$ ) 
Due to the severe deterioration of the Kynar targets when bombarded by the incident beam of alpha particles, it was found unnecessary to measure the initial thickness of each Kynar target. Instead, each target was constantly monitored by a fixed surface-barrier-type detector mounted in the spectrograph scattering chamber. These target-monitor spectra were later normalized by comparing them with a similar spectrum from a Teflon foil of known thickness.

The angular distributions corresponding to alpha-particle reactions leading to the ground and first five excited states in the $F^{19}$ nucleus were measured at $5^{\circ}$ intervals in the range of laboratory scattering angles between $15^{\circ}$ and $155^{\circ}$. The reaction information was then read from the nuclear-track emulsions and these data were reduced to absolute differential cross sections expressed in the center-of-mass system.

The angular distributions for the ground state $\left(1 / 2^{+}\right)$and the $0.1970-$ $\mathrm{MeV}$ state $\left(5 / 2^{+}\right)$span the common center - of -mass angular range $18^{\circ}$ to $160^{\circ}$. These angular distributions display a strong diffraction-like pattern at the forward angles, and their behavior is consistent with the predictions of the Blair phase rule, 1. e., the oscillations of the $0.1970-\mathrm{MeV}$ state are out of phase with the oscillations of the ground state. ${ }^{3)}$ Furthermore, at the back angles these angular distributions display the washed-out diffraction-like pattern which has been observed in the angular distributions of other light odd-A nuclei. ${ }^{4)}$ It is believed that the probability for a transition to the $0.10987-\mathrm{MeV}$ state $\left(1 / 2^{-}\right)$of $\mathrm{F}^{19}$ is very small since no tracks corresponding to this transition were found in the nuclear-track emulsions.

The common center-of-mass angular range for the angular distributions corresponding to the excited states in the second triplet of levels in the $F^{19}$ nucleus is limited to the region between $18^{\circ}$ and $98^{\circ}$. This angular 
interval is restricted because of the simultaneous occurrence of dominant interferring groups of tracks arising from reactions involving the target contaminant nuclei. The three angular distributions for this second triplet of levels all show a strong diffraction-like pattern at the forward angles and appear to be consistent with predictions of the Blair phase rule.

Four-parameter optical-model analyses utilizing both the SaxonSaxon and the Saxon-Saxon-derivative forms for the nuclear potential have been performed on the elastic-scattering data. The various sets of these optical-model parameters have now been used in a distorted-wave Bornapproximation (DWBA) analys is to describe the collective excitation, i. e., the $5 / 2^{+}$and the $3 / 2^{+}$levels, in the $F^{1.9}$ nucleus. A DWBA analysis utilizing two different cluster descriptions of the $\mathrm{F}^{19}$ nucleus is currently in progress.

1. The spin and parity assignments are taken from J. P. Allen, A. J. Howard, D. A. Bromley, and J. W. Olness, Phys. Rev. 140, B 1245, (1965). The excitation-energy assignments are taken from T. Lauritsén " and F. Ajzenberg-Selove, Nuclear Data Sheets-Energy Levels of Light Nuclei, May 1962 (National Academy of Sciences-National Research Council, Washington, D. C., (1962)).

2. The Kynar powder was made available through the courtesy of the Pennsalt Chemicals Corporation, Philadelphia, Pennsylvania.

3. J. S. Blair, Phys. Rev. 115, 928 (1959)

4. B. T. Lucas, S. W. Cosper, and O. E. Johnson, Phys. Rev. 144, 972 , (1966). 


\section{COULOMB EXCITATION STUDIES}

\section{(TANDEM VAN DE GRAAFF ACCELERATOR)}

\section{A. Static Quadrupole Moment of the $2^{+}$State in $\mathrm{Cd}^{\mathrm{I}} 14$}

\section{Determined by Coulomb Excitation}

\section{G. Schilling, R. P. Scharenberg, and J. W. Tippie}

We have measured the reorientation effect and thus the quadrupole moment of the $0.558-\mathrm{MeV}^{+}$state in $\mathrm{Cd}^{114}$ by observing the angular distribution of $25-\mathrm{MeV}$ oxygen ions inelastically scattered off $\mathrm{Cd}^{114}$ nuclei. The shape of the angular distribution gives conclusive evidence of the presence of the reorientation effect. The scattered ions were detected in coincidence with the de-excitation gamma radiation over an angular range from $50^{\circ}$ to $160^{\circ}$ in the laboratory.

The advantages of this technique are these: (1) Only a single type of projectile is required. Consequently possible penetration by the lighter projectile is eliminated. (2) With our particular geometry, favorable counting rates are obtained by operating several detectors simultaneously. (3)'Because of the favorable counting rates, the experiment can be executed at lower bombarding energy, where the uncertainties in the theoretical interpretation are considerably reduced. (4) The angular distribution can aid in distinguishing the reorientation effect from excitation via the giant dipole resonance.

The separated-isotope $\mathrm{Cd}^{114}$ targets were prepared by vacuum depositing $200-\mu \mathrm{g} / \mathrm{cm}^{2} \mathrm{Cd}^{114}$ metal on a $75-\mu \mathrm{g} / \mathrm{cm}^{2} \mathrm{Cu}$ backing. A thin $\left(20-\mu \mathrm{g} / \mathrm{cm}^{2}\right)$ $\mathrm{Cu}$ evaporation shield was deposited on top of the $\mathrm{Cd}$ metal. The enrichment was $97 \%$. 
The 25-MeV oxygen ions were produced by the Argonne National Laboratory tandem acceler ator.

Following Alder et al. , 1) the ratio of inelastic to elastic Coulomb scattering is given by

$$
\frac{d \sigma_{i} / d \Omega}{d \sigma_{e} / d \Omega}=P(\theta),
$$

where $P(\theta)$ is the probability of excitation. We can apply this expression to the experiment if the variation of the gamma-ray angular distribution with ion scattering angle $\theta$ is taken into account. This is possible because of the insensitivity of the gamma-ray angular distribution to the reorientation effect. We have measured the relative photopeak efficiency with respect to the symmetry axis of the crystal. This efficiency is folded with the gamma-ray angular distribution obtained from the semi-classical theory. Thus, a "gating efficiency" can be determined for the various scattering angles. In addition we measured the gamma-ray angular distribution with respect to the incident beam direction and found it unperturbed.

The experimental excitation probabilities are shown in Fig. 1. A marked deviation from first-order Coulomb excitation theory is evident.

We have investigated the magnitude of higher order effects compared to the reorientation effect. We used the semiclassical theory and the $B(E 2)$ values measured by Stelson and Mc-Gowan. 2) We find: (1) The population of the $0.558-\mathrm{MeV} 2^{+}$state is changed by only $0.15 \%$ by de-excitation from higher states. (2) The uncertainty in theoretical interpretation of the reorientation effect due to virtual excitation of higher levels is insensitive to the magnitude of the reorientation effect and varies rapidly with the bombarding energy. For $25-\mathrm{MeV}$ oxygen ions the effect of the higher levels introduces a 
$\pm 1 \%$ uncertainty in the excitation probability at $162^{\circ}$ in the center-of-mass system.

We have fitted the experimental data with the function

$$
P(\theta)=A(\theta)+M_{22} B(\theta) \text {, }
$$

where $A(\theta)$ and $B(\theta)$ are obtained from the two-level semiclassical theory using $M_{22}$ values which straddle the data. A maximum-likelihood fit gives $M_{22}=0.85 \pm 0.15$. If we include the uncertainties introduced by the higher levels $M_{22}=0.85 \pm 0.25$. Since

$$
-e Q_{22}=\frac{4}{5}\left(\frac{2 \pi}{7}\right)^{\frac{1}{2}} M_{22}
$$

we obtain

$$
Q_{22}=-0.64 \pm 0.19 \times 10^{-24} \mathrm{~cm}^{2}
$$

for the quadrupole moment of the $0.558-\mathrm{MeV}^{+}$state of $\mathrm{Cd}^{114}$, in agreement with ratio measurements. This value of the quadrupole moment is in striking contrast with the vanishingly small quadrupole moments predicted by the pure vibrational model.

This analysis neglects the possibility of excitation via the giant dipole resonance, which is believed to be small. ${ }^{3)}$ The observed angular distribution is in good agreement with the reorientation-effect mechanism and suggests experimentally that the giant dipole resonance effects are very small.

The results of this investigation have been published in Physical Review Letters, 19, 318, (1967).

1. K. Alder, A. Bohr, T. Huus, B. Mottelson, and A. Winther, Rev. Mod. Phys. 28, 432, (1956).

2. P. H. Stelson and F. K. McGowan, Phys. Rev. 121, 209, (1961); F. K. McGowan et al., Bull. Am. Phys. Soc. 9, 107, (1964).

3. N. McDonald, Phys. Letters 10, 334, (1964). 


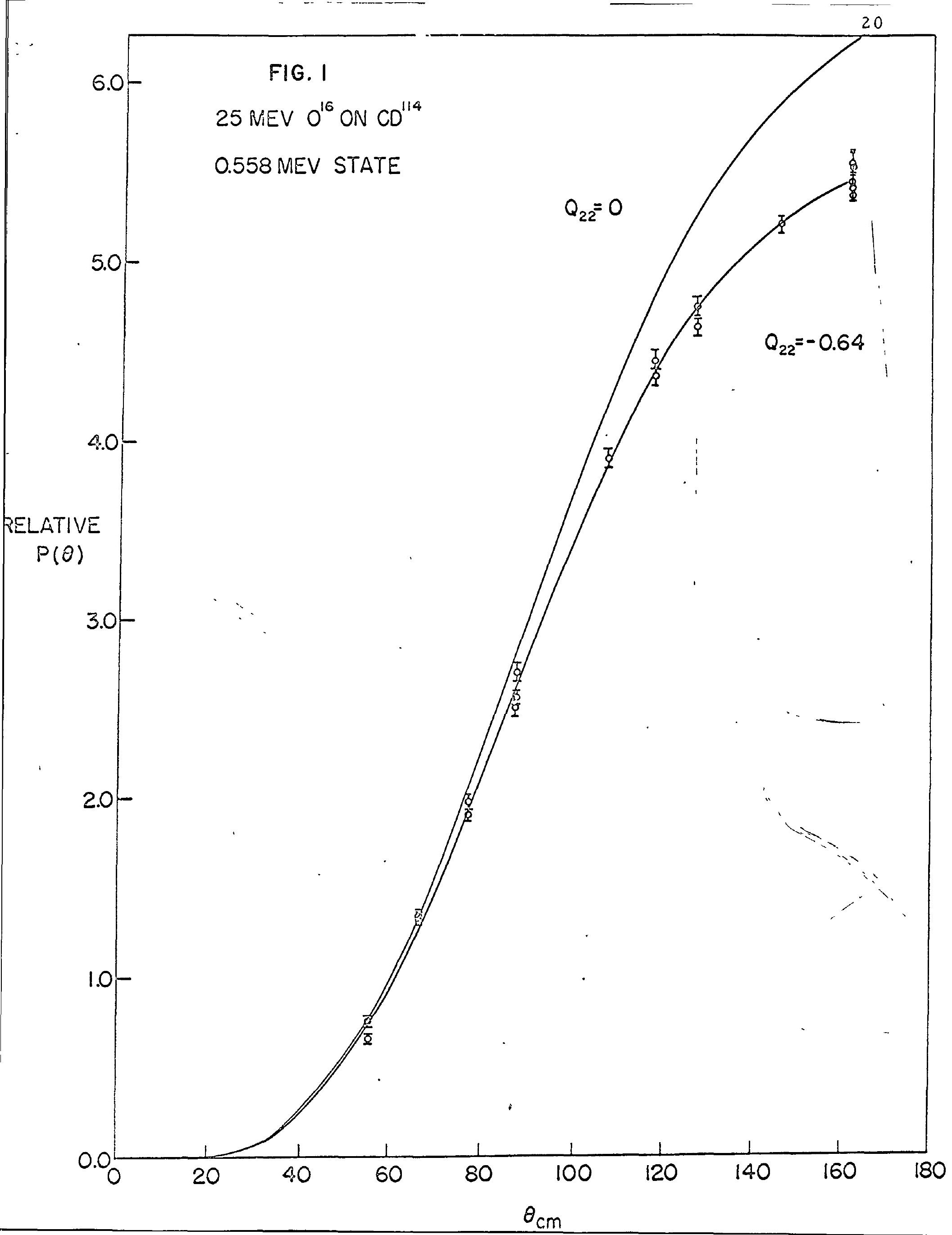


B. Static Quadrupole Moment of the First Excited $2^{+}$State $12 \mathrm{Fe}^{56}$

R. P. Scharenberg and J. W. Tippie

Preliminary measurements of the quadrupole moment of the 0.858 $\mathrm{keV} 2^{+}$state in $\mathrm{Fe}^{56}$ have been made with the $25 \mathrm{MeV} \mathrm{O}{ }^{16}$ beam from the Argonne Tandem. Least squares fits to two experiments yield $M_{22}=$ $0.62 \pm .10$ and $M_{22}=0.43 \pm .08$. Figure 2 shows the results of the latter measurement with the fitted curve. These preliminary experiments yield a measured quadrupole moment of $-.39 \pm .05$ barns. Effects due to higher states have been neglected thus far and are believed to be small. The above experiments were performed on self supportıng natural $\mathrm{Fe}$ targets of $150-300 \mu \mathrm{g} / \mathrm{cm}^{2}$ thickness prepared by vacuum deposition techniques.

The data points at $166^{\circ}$ ( $\left.C M\right)$ in the above data have tended to be somewhat lower than theory would predict. This has resulted in rather poor quality fits when compared with that expected from statistics. This phenomena is currently being investigated. The electronics is currently being checked for possible instrumental effects. A self checking feature is being incorporated which will allow continuous checking during an actual measurement. If it is assumed that this is a real effect two other possibilities have been suggested. There is the possibility of barrier penetration by the projectile. This, however, does not appear likely as at $25 \mathrm{MeV}$ the distance of closest approach is about $15.4 \mathrm{~F}$ while the sum of the two nuclear radii is about $9.0 \mathrm{~F}$. Thus in the worst case of a head-on collision the nuclear surfaces are about 6.4 F apart. Furthermore, measurement of the elastic ion angular distribution does not indicate any significant deviation from 
Rutherford scattering. The last possibility is that of hexadecapole mixing. Preliminary calculations by Walter Johnson of Notre Dame University (private communication) indicate that hexadecapole mixing could account for a several percent effect in the excitation probability at the extreme back angles. 


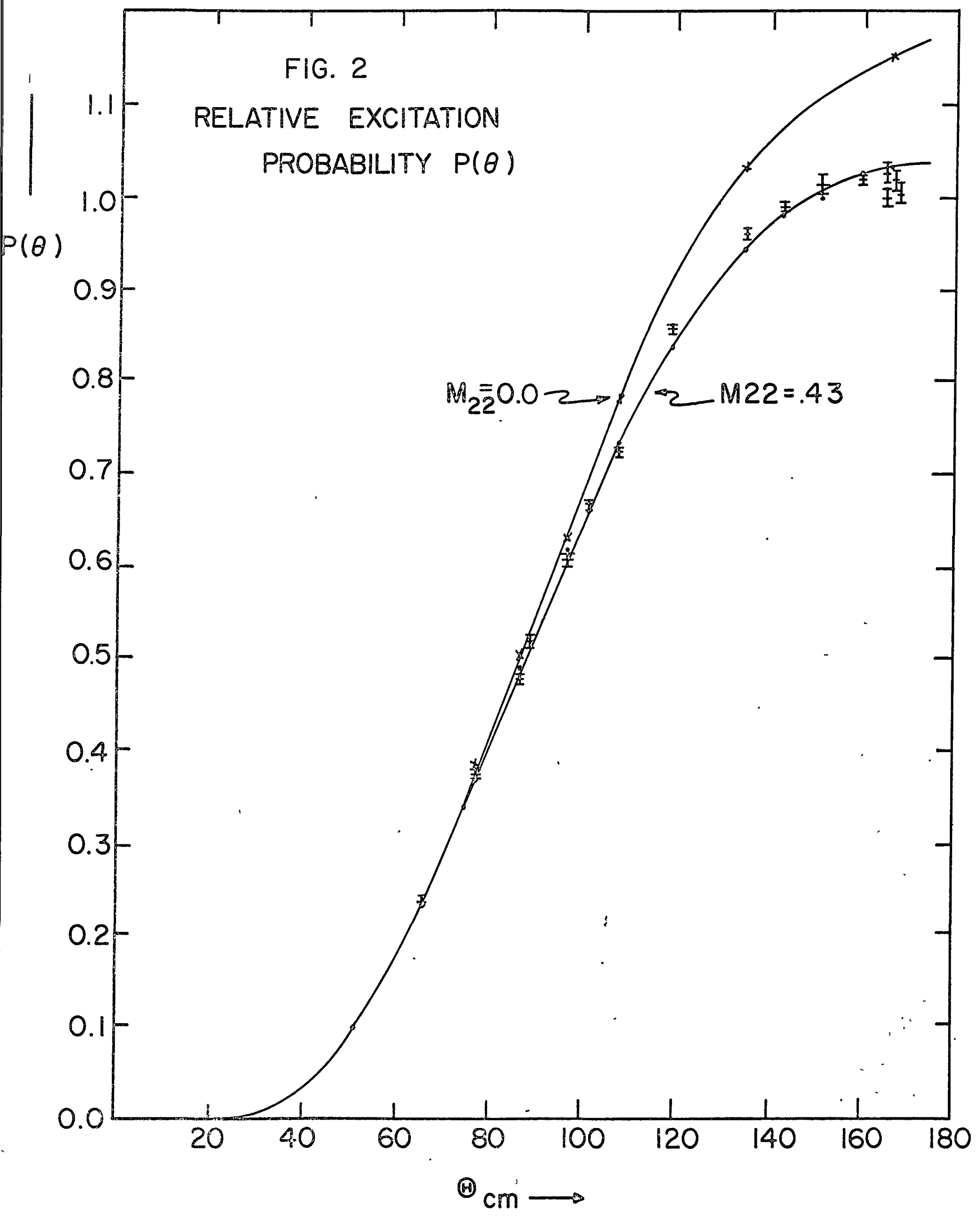




\section{ANGULAR CORRELATION STUDIES :}

\section{A. Search for Higher Order Effects in Allowed $\beta$ Transitions}

Z. W. Grabowski, R. S. Raghavan, and R. M. Steffen

The $\beta-\gamma$ directional correlations of several allowed $\beta$ transitions with unusually large $\mathrm{ft}$ values $(\log \mathrm{ft}>7)$ were measured. Effects of secondforbidden $\beta$ components are expected to manifest themselves in small observable anisotropies if second-forbidden matrix elements are not reduced appreciably by those nuclear-structure effects that cause the reduction of the allowè. matrix elements. The experimental results for the anisotropy factor $A_{22}{ }^{\prime}(W)$ in the $\beta-\gamma$ directional correlation function $W(\theta)=1+A_{22}(W) P_{2}(\cos \theta)$ are:

$$
\begin{aligned}
& \mathrm{Na}^{22}\left(\mathrm{~W}_{0}=1.69, \log \mathrm{ft}=7.4\right) ; \mathrm{A}_{22}(\mathrm{~W}=1.8)=-0.001 \pm 0.0005 ; \\
& \mathrm{Co}^{56}\left(\mathrm{~W}_{0}=3.88, \log \mathrm{ft}=8.6\right), \mathrm{A}_{22}(W=3.3)=-0.001 \pm 0.0003 ; \\
& \mathrm{Sb}^{124}\left(\mathrm{~W}_{0}=2.23, \log \mathrm{ft}=7.7\right), \mathrm{A}_{22}(\mathrm{~W}=2.0)=+0.0002 \pm 0.0003 ; \\
& \mathrm{Tb}^{160}\left(\mathrm{~W}_{0}=2.1, \log \mathrm{ft}=8.1\right), \mathrm{A}_{22}(\mathrm{~W}=1.9)=+0.013 \pm 0.007 ; \\
& \mathrm{Cs}^{134}\left(\mathrm{~W}_{0}=2.3, \log \mathrm{ft}=8.9, \mathrm{~A}_{22}(\mathrm{~W}=1.9=+0.001 \pm 0.003 .\right.
\end{aligned}
$$

Except for the highly deformed $\mathrm{Tb}^{160}$, no evidence of interference effects with second-forbidden $\beta$ components was found, indicating that whatever the mechanism that reduces the allowed matrix elements it somehow also reduces the second-forbidden matrix elements. The small anisotropy found in $\mathrm{Tb}^{160}$ may be the result of some $K$ selection rule effect or may be caused by a competing forbidden $\beta$ transition. Evidence for the existence of such a $\beta$ transition (which, however, is very difficult to fit into the established $\mathrm{Tb} 160$ decay scheme) is given.

The results of this investigation have been published in The Physical Review, 139. B24 (1965). 


\section{B. Higher Order Effects in $\beta-\gamma$ Correlations Involving}

Allowed $\beta$ Transitions

S. Cipolla, Z. W. Grabowski, H. M. Nasir, and R. M. Steffen

Higher order effects in allowed $\beta$ transitions may be generated by the interference of the allowed Gamow-Teller components with secondforbidden components. In strongly hindered $\beta$ transitions, this interference effect is expected to give rise to $\beta-\gamma$ directional correlations with measurable anisotropies. The $\beta-\gamma$ directional correlations of the hindered. allowed $\beta$ transition $(\log \mathrm{ft}>8)$ in the deformed nuclei $\mathrm{Eu}^{152}$, and $\mathrm{Tb}^{160}$ were studied. The $\beta$-correlation factor $\mathrm{A}_{2}{ }^{\beta}$, which characterizes the $\beta$ transition in the expression for the $\beta-\gamma$ directional correlation $W(\theta)=$ $1+A_{2}{ }^{\beta} A_{2}{ }^{\gamma} P_{2}(\cos \theta)$, was found to be vanishingly small in all cases, except that of $\mathrm{Tb}^{160}$. The results are :

$A_{2}{ }^{\beta}=+0.017 \pm 0.012$ for the $0.70-\mathrm{MeV} \beta$ transition of $\mathrm{Eu}^{152}(\log \mathrm{ft}=10.6)$; $A_{2}{ }^{\beta}=+0.016 \pm 0.020$ for the $0.20-\mathrm{MeV} \beta$ transition of $\mathrm{Eu}^{152}(\log \mathrm{ft}=9.6)$; $A_{2}{ }^{B}=0.007 \pm 0.006$ for the $0.58-\mathrm{MeV} \beta$ transition of $E^{154}(\log \mathrm{ft}=10.0)$; $A_{2}{ }^{B}=+0.041 \pm 0.015$ for the $0.566-\mathrm{MeV} \beta$ transition of $\mathrm{Tb}^{160}(\log \mathrm{ft}=8.1)$. A summary of $\beta-\gamma$ directional correlation measurements on allowed $\beta$ transitions is presented and the implications of the results are discussed. In most cases the experimental results suggest that the effects that cause the reduction of the allowed matrix elements are also operative in reducing the sec ond-forbidden matrix elements.

The results of this investigation have been published in The Physical Review, 146, 877 (1966).

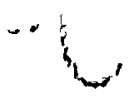




\section{Study of Second Excited $2^{+}$States of Some Even-Even Nuclei by Beta-Gamma Angular Correlations}

R. S. Raghavan, Z. W. Grabowski, and R. M. Steffen

Beta-gamma directional correlations of several first-forbidden $\beta$ transitions leading to second excited states of even-even nuclei ( $\beta_{2}$ transitions) have been studied. The energy-dependence of the $\beta$ directional correlation factor $A_{2}(\beta)$ in the $\beta-\gamma \operatorname{correlation}$ function $W(\theta)=$ $1+A_{2}(\beta) A_{2}(\gamma) P_{2}(\cos \theta)$ has been measured for the $\beta_{2}$ transitions of $A s^{76}$, $\mathrm{Sb}^{122}, \mathrm{I}^{126}, \mathrm{Sb}^{124}$, and $\mathrm{La}^{140}$, and the reduced $\beta$ coefficient $\mathrm{R}_{2}(W)=$ $A_{2}(\beta) /\left(\lambda_{2} p^{2} / W\right)$ was determined. In all cases $R_{2}(W)$ was found to be very different from $R_{1}(W)$, the reduced $\beta$ factor describing the $\beta_{1}$ transition to the first excited state of the daughter nucleus. The results indicate that the relative magnitudes of the nuclear $\beta$-matrix elements in the $\beta_{1} \operatorname{tran-}$ sition and in the $\beta_{2}$ transition are significantly different, although the ft values are very similar. The implication of these experimental results for the structure of first and second excited states of even-even spherical nuclei is discussed.

The results of this investigation have been published in the Physical Review, 139, B 1, (1965). 
D. Nuclear Matrix Elements in the First-Forbidden 2. 2-MeV $\beta$ Transition of La 140

R. M. Singru, P. C. Simms, and R. M. Steffen

The angular dependence of the circular polarization of the $1.6-$ $\mathrm{MeV} \gamma$ radiation following the $2.2-\mathrm{MeV} \beta$ transition of $\mathrm{La}^{140}$ has been measured. The analysis of these data, together with $\beta-\gamma$ directionalcorrelation and spectrum-shape data, was made using exact electron wave functions and taking into consideration finite-nuclear-size effects. The nuclear beta matrix elements determined in this manner show that the deviations of the $2.2-\mathrm{MeV} \quad \beta$ transition from the Coulomb approximation is caused by a cancellation effect of the vector-type matrix elements and not by a selection-rule effect. The experimentally determined ratio of the $\int i \vec{\alpha}$ and the $\int \vec{r}$ matrix elements does not agree well with the value predicted by Fujita on the basis of the conserved-vector-current theory. The discrepancy may be due to the presence of higher order effects in the 2.2$\mathrm{MeV} \beta$ transition of $\mathrm{La}^{140}$.

The results of this investigation have been published in the Physical Review, $141,1078,(1966)$. 


\section{E. Beta-Gamma Circular Polarization Correlation of
Z. W. Grabowski, R. S. Raghavan and R. M. Steffen}

The angular dependence of the circular polarization of the $566 \mathrm{keV}$ gamma-radiation following the first-forbidden $1400 \mathrm{keV} \beta$-transition of $\mathrm{Sb}^{122}$ has been studied. The $\beta-\gamma$ circular polarization correlation is consistent with $a \cos \theta$ dependence. The value of the $\beta-\gamma \operatorname{circular}$ polarization anisotropy factor $A_{1}(\beta)$ in the correlation function $W(\theta, \tau)=1+\tau A_{1}(\beta) A_{1}(\gamma) x$ $P_{1}(\cos \theta)+A_{2}(\beta) A_{2}(\gamma) P_{2}(\cos \theta)$ is $A_{1}(\beta)=(0.09 \pm 0.04) p / W$. The data confirm that shape and the angular correlations of the $1400 \mathrm{keV} \quad \beta$-transition of $\mathrm{Sb}^{122}$ are well described by the Coulomb approximation. The ratio of scalar type to vector type matrix element components in this $\beta$-transition is (in Kotani's notation $): x_{1}=\left[C_{A} \int i \gamma_{5}+(\alpha Z / 2 R) C_{A} \int \vec{\sigma} \cdot \vec{x}\right] /\left[-C_{V} \int i \vec{\alpha}-(\alpha Z / 2 R) x\right.$ $\left.\left(C_{A} \int i \vec{\sigma} \times \vec{r}-C_{V} \int \vec{r}\right)\right]=13_{-5}^{+10}$ or $x_{2}=0.29 \pm 0.04$. The second solution can be excluded on the basis of angular distribution data from oriented nuclei. The $\beta-\gamma$ circular polarization correlation results obtained, make it possible to reduce the number of solutions obtained by Pipkin et al. for the six nuclear matrix elements which enter into the $2^{-} \rightarrow 2^{+} 1400 \mathrm{keV} \beta$-transition of Sb ${ }^{122}$. The results of this investigation have been published in Nuclear Physics, $\underline{70}$, $170(1965)$. 
F. Energy Dependence of Beta-Gamma Circular Polarization

P. C. Simms, D. Ohlms, and J. Bosken

The instrument to be used in this experiment has been completed, and calibration measurements are in progress. A circular polarization analyzer magnet was available when the project was started. An automatic control for the magnet has been built which will reverse the magnetic field or degauss the magnet. The control unit can be operated manually or op-. erated automatically from the data system master control. A new set "of lead collimators was built to optimize the circular polarization analysis for the isotopes which we plan to study. A computer program was written to calculate the efficiency of the circular polarization analyzer.

A new vacuum chamber has been built so that special beta detectors with good energy resolution can be used. The beta detectors are well-type plastic scintillators which minimize back scattering. These detectors mounted on RCA 8575 P.M. tubes give $9 \%$ energy resolution for $1 \mathrm{MeV}$ electrons. The angular dependence of the beta-gamma circular polarization can also be measured with this chamber.

The most important feature of the new electronic system developed for this experiment is that data $c$ an be recorded at a very high rate and prepared for direct computer processing. The system is also very stable so that systematic errors are minimized. Commercial high-voltage stabilizers are used on both the beta and gamma scintillation detectors. The beta detector presented a problem for the stabilizer system since there is no sharp peak in a beta spectrum for the stabilizer to lock on. To overcome this problem, a small NaI scintillator which contains an alpha source was attached to the beta detector P.M. tube. The scintillator serves as a light pulser which gives a 
signal approximately 30 times larger than the beta particles. The output of the beta detector goes to two D. D. L. amplifiers. One amplifier is set for high gain and used $100 \mathrm{nsec}$ delay lines. This amplifier can feed the beta energy analysis system at counting rates in excess of $1 \mathrm{MHz}$. Of course the large NaI pulse overloads this amplifier quite badly. A special linear amplifier was developed for high counting rate and overload conditions. (See V -C of this report). The other linear amplifier passes the NaI pulses to the stabilizer system. Since the beta pulses are so much smaller than the NaI pulses, the very high beta counting rate does not effect the stabilizer.

Fast timing pulses from the beta and gamma detectors are taken to a high rate, good resolution time-to-amplitude converter. This time converter is used with two single-channel analyzers to record the true and accidental counting rates at the same time. With this technique very strong sources and high counting rates can be used. In fact for a given source strength, the best statistical results are obtained with a one-to-one true-to-chance ratio.

In addition to the data stored in the multichannel analyzer, all of the other important counting rates are recorded by an automatic scaler system. All of the data is automatically punched on paper tape for computer processing.

Measurements on $\mathrm{Co}^{60}, \mathrm{Rb}^{86}$, and $\mathrm{La}^{140}$ are being performed. $\mathrm{Co}^{60}$ is being studied to provide an accurate test of the theoretical prediction that the circular polarization is proportional to $\mathrm{V} / \mathrm{C}$. The measurement on $\mathrm{Rb}^{86}$ and $\mathrm{La}^{140}$ will be combined with other data to make an accurate determination of the nuclear matrix elements for the se two isotopes. It is expected that the result will be sufficiently accurate so that the predictions of CVC theory for the ratio of nuclear matrix elements can be tested. 


\section{G. Transverse Polarization of $\mathrm{K}$-Conversion Electrons}

Following the $\beta$ Decay of $\mathrm{Au}^{198}$

R. L. Rasera and R. M. Steffen

The degree of transverse polarization $P_{\|}(\theta)=K_{K}(v / c) \sin \theta$ of the K-conversion electrons following the beta decay of $\mathrm{Au}{ }^{198}$ has been mea- $\therefore$ sured with a view to the determination of the particle parameter $b_{1} \|_{1}(E 2)$. The experimental result was obtained using a lens spectrometer to focus the conversion electrons on a thin gold foil, and measuring the Mott-scat-. tering asymmetry $\delta$ of the counts in coincidence with those detected in a beta detector placed opposite the source at an angle $\theta$ of $90^{\circ}$ with the spectrometer axis. The measured asymmetry of $\delta=-0.044 \pm 0.013$ yields a value of $\mathrm{K}_{\mathrm{K}}=+0.43 \pm 0.17$, with all instrumental corrections applied. Given the beta-decay angular correlation coefficient $A_{1}(B)$ known from beta circularly polarized gamma correlations, the particle parameter is found to be $\mathrm{b}_{1 \|}(\mathrm{E} 2)=-1.0 \pm 0.4$. This value, although in disagreement with previous measurements, agrees with the value calculated by Becker and Rose.

The results of this investigation have been published in The Physical Review, 140, BI177 (1965) 


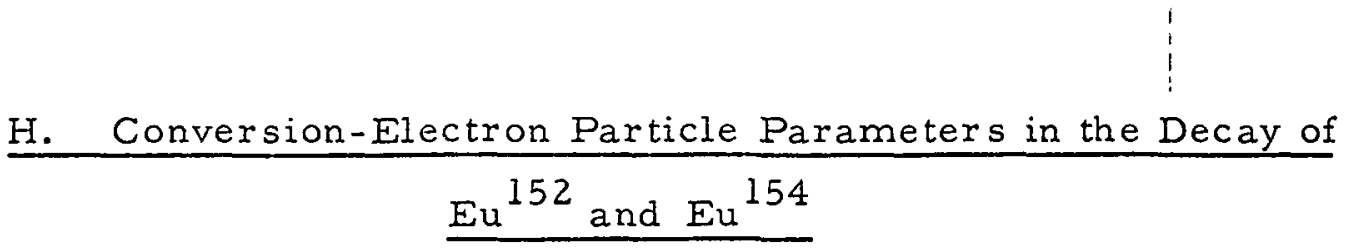

H. M. Nasir, Z. W. Grabowski, and R. M. Steffen

The internal conversion-electron particle parameters of several fast low-energy E2 transitions in the deformed nuclei $\mathrm{Sm}^{152}$ and Gd 154 were determined by comparing the $e^{-}-\gamma$ directional correlations and $\gamma-\gamma$ directional correlations displayed by the same sources. Special attention was paid to eliminating the effects of scattering in the source, and the corrections caused by the presence of extranuclear perturbations of the directional correlations were carefully studied. The results are : 0.122-MeV transition in $\mathrm{Sm}^{152}: \mathrm{b}_{2}(\mathrm{E} 2, \mathrm{~K})=1.60 \pm 0.05, \mathrm{~b}_{2}\left(\mathrm{E} 2, \mathrm{~L}^{-}\right)=1.03 \pm 0.05$; $0.245-\mathrm{MeV}$ transition in $\mathrm{Sm}^{152}: \mathrm{b}_{2}(\mathrm{E} 2, \mathrm{~K})=1.72 \pm 0.07 ; 0.344-\mathrm{MeV}$ transition in $\mathrm{Gd}^{154}: \mathrm{b}_{2}(\mathrm{E} 2, \mathrm{~K})=1.57 \pm 0.13 ; 0.123-\mathrm{MeV}$ transition in Gd ${ }^{154}$ : $\mathrm{b}_{2}(\mathrm{E} 2, \mathrm{~K})=1.37 \pm 0.07$. The two particle parameters of the $0.122-\mathrm{MeV}$ ground-state transitions in $\mathrm{Sm}^{152}$ are about $15 \%$ lower and that of the $0.123-\mathrm{MeV}$ ground state-transition in $\mathrm{Gd}^{154}$ is $25 \%$ lower, than the theoretical predictions for finite-size nuclei. The particle parameters of the $0.245-\mathrm{MeV}$ and of the $0.344-\mathrm{MeV}$ transitions agree with the theoretical values within experimental errors.

The results of this investigation have been published in The Physical Review, 162, $1118(1967)$. 
I. Conversion-Electron Gamma Directional Correlations in $\underline{\operatorname{Tm}^{169} \text { and } L u^{175}}$

H. M. Nasir, Z. W. Grabowski, and R. M. Steffen

The angular correlation table of the lens-spectrometer has been modified to accommodate a moveable cryostat and a Ge(Li) gamma detector. Measurements of $e^{-}-\gamma$ directional correlations have been started, where the high resolution of the Ge(Li) detector is essential.

The particle parameters of the conversion electrons are computed from the measured $e^{-}-\gamma$ directional correlation and from the corresponding $\gamma-\gamma$ directional correlation. The particle parameters of $\gamma$-transitions in $\mathrm{Tm}^{169}$ and $\mathrm{Lu}^{175}$ are being studied.

The study of particle parameters of the conversion electrons in the Im ${ }^{169}$ transition is of particular interest, since the nucleus is strongly deformed. Also the influence of penetration effects may be investigated in this manner.

The results for the directional correlations of the $\gamma(177)-K(131)$ cascade in $\operatorname{Tm}^{169}$ are:

$$
\begin{aligned}
& \text { A. }, W(\theta)=1+(0.482 \pm 0.014) \mathrm{P}_{2}(\cos \theta) \\
& \text { B. } \quad W(\theta)=1+(0.496 \pm 0.008) \mathrm{P}_{2}(\cos \theta)
\end{aligned}
$$

The result $A$ was obtained with the Ge(Li) gamma detector; the result $B$ was obtained with the $\mathrm{NaI}(\mathrm{T} l)$ detector.

The $\gamma(177)-\gamma(131)$ directional correlation, as measured with two Ge(Li) detectors is:

$$
W(\theta)=1+(0.253 \pm 0.008) P_{2}(\cos \theta)+(0.036 \pm 0.010) P_{4}(\cos \theta)
$$


From these measurements the $\mathrm{K}$-particle parameter of the $131 \mathrm{keV}$ transition was computed:

$$
b_{2}(131 ; K)=1.93 \pm 0.10
$$

This value is in fair agreement with the previously reported results within limits of error, but it seems to indicate a somewhat larger particle parameter than earlier measurements. 1) The theoretical value for a pure E2 transition is : $\mathrm{b}_{2}(\mathrm{E} 2 ; \mathrm{K})=1.85$.

According to the $\gamma-\gamma$ directional correlation measurements, the co- . efficient of the $\mathrm{P}_{4}(\cos \theta)$ term is of the order of a few percent. The theoretical $b_{4}(E 2 ; K)$ particle parameter is $b_{4}(E 2 ; K)=-1$. The search for a $\mathrm{P}_{4}(\cos \theta)$ term in the $\mathrm{e}^{-}-\gamma$ directional correlation is in progress.

The particle parameter of the $282 \mathrm{keV}$ transition in Lu 175 " which presumably is an El transition with a small M2 admixture, has been mèasured before. ${ }^{2,3)}$ Two rather different values of the particle parameter were reported $b_{2}(282, K)=+0.06 \pm 0.12^{I}$ and $b_{2}(282, K)=+0.27 \pm 0.06^{2}$. Both these values disagree strongly with the theoretical value, $b_{2}(E I ; K)=$ - 1.52, which does not include dynamic penetration effects.

The $\mathrm{Yb}^{175}$ sources were prepared by means of an isotope separator, hence the corrections for scattering effects in the extremely thin sources were negligibly small. The result of the $e^{-}-\gamma$ directional correlation measurement on the $K(282)-\gamma(114)$ cascade is:

$$
W(\theta)=1+(0.10 \pm 0.05) P_{2}(\cos \theta)
$$

The measurement of the corresponding $\gamma-\gamma$ directional correlation is in progress. 
If one takes the $\gamma-\gamma$ directional correlation results of Thun et ${ }^{2}{ }^{2}$ ), the particle parameter extracted from the present measurements is:

$$
\mathrm{b}_{2}(282 ; \mathrm{K})=+0.04 \pm 0.02
$$

The result indicates, that there is definitely a large discrepancy with theory. The investigation of this discrepancy is in progress.

1. Z. W. Grabowski, J. E. Thun, M. S. El-Nesr, and W. K. Hamilton, Z. Physik 167, 111 (1962).

2. J. E. Thun, Z. Grabowski, M. S. El-Nesr, Nuclear Physics 29, 1 (1962).

3. J. E. Thun, Nuclear Physics A91, 653 (1967).

J. $K, I$ and $M$ Internal Conversion Parameters of M4 Transitions

D. L. Coppage and R. M. Steffen

In a study of the internal conversion parameters of M4 transitions, attention has been given to the M4 transitions of the isotopes $\mathrm{Bi}^{207}, \mathrm{Sn} 117^{\prime \prime}$, “ and $\mathrm{Hg}^{197}$. The measurements on the electron spectra have been made ," ' " using a cooled lithium drifted silicon detector, which has a normal resolution of about $6 \mathrm{keV}$ FWHM. In the case of heavy elements this is usually sufficient to resolve the M-line from the L-line and to permit a relatively clean measurement of the $M$-line conversion parameters The logic circuits of the system are of a standard type utilizing timing single channel analyzers and a single fast coincidence circuit for each channel. The measurements on the $13 / 2(1064 \mathrm{M} 4) 5 / 2(570 \mathrm{E} 2) \mathrm{I} / 2$ cascade of $\mathrm{Bi}^{207}$ are almost complete. The angular correlation functions of this cascade determined so far are: 
1. $1064 \mathrm{~K}-570 \mathrm{\gamma}$

$$
\begin{aligned}
& W(\theta)=1+0.237(3) P_{2}(\cos \theta)-0.034(4) P_{4}(\cos \theta) \\
& W(\theta)=1+0.239(6) P_{2}(\cos \theta)+0.012(8) P_{4}(\cos \theta) \\
& W(\theta)=1+0.267(6) P_{2}(\cos \theta)-0.006(7) P_{4}(\cos \theta) \\
& W(\theta)=1+0.22(2) P_{2}(\cos \theta)+0.006(30) P_{4}(\cos \theta) \\
& W(\theta)=1+0.236(4) P_{2}(\cos \theta)+0.022(4) P_{4}(\cos \theta)
\end{aligned}
$$$$
\text { 2. } 1064 \mathrm{~L}_{\text {Tot }^{-570 \gamma}}
$$$$
\text { 3. } 1064 \gamma-570 \mathrm{~K}
$$$$
\text { 4. } 1064 \gamma-570 L_{\text {Tot }}
$$$$
\text { 5. } 1064 \gamma-570 \gamma
$$

The angular correlation coefficients for the $1064 \gamma-570 \gamma$ cascade after being corrected for a contribution from the $1771 \gamma-570 \gamma$ cascade indicate some small mixture of E5 in the predominately M4 $1064 \mathrm{keV}$ transition in agreement with a recent report. ${ }^{1)}$ The set of data for the $1064 \mathrm{keV}$ and $570 \mathrm{keV} M$-lines is still incomplete.

The data analysis for the $11 / 2(159 \mathrm{M} 4) 3 / 2(158 \mathrm{MI}) 1 / 2$ cascade in. $\mathrm{Sn}^{117}$ is still in progress. Preliminary results for the angular correlation functions determined so far are as follows:

$$
\begin{array}{ll}
\text { 1. } 159 K-158 \gamma & W(\theta)=1-0.143(4) P_{2}(\cos \theta) \\
\text { 2. } 159 \gamma-158 \gamma & W(\theta)=1-0.139(8) P_{2}(\cos \theta)
\end{array}
$$

The last angular correlation function which is to be determined is the 159( L $_{\text {Tot }}+M_{\text {Tot }}$ ) - 158y cascade for which the $M$-line of the M4 transition. is not resolved.

The last case under consideration is the determination of the internal conversion parameters for the well resolved $K, L$, and $M$ electrons in the M4 transition in the $13 / 2(165 \mathrm{M} 4) 5 / 2(133 \mathrm{E} 2) 1 / 2$ cascade in $\mathrm{Hg}^{197}$ for which the experiment is in progress.

1. P. Kleinheinz, et al., Nuclear Physics A93, 63 (1967). 
K. Beta-Gamma Directional Correlations in the Decay' of $\mathrm{Sb}^{124}$

S. Cipolla and R. M. Steffen

The $\beta-\gamma$ directional correlation of the $2.39 \mathrm{MeV} \beta$ transition with the $0.063 \mathrm{MeV}$ gamma transition is well known. A clean measurement of the $1.59 \mathrm{MeV} \beta-0.722 \gamma$ and $1.68 \mathrm{MeV} \beta-0.645 \gamma$ directional correlations, however, was not possible with $\mathrm{NaI}(\mathrm{T} l)$ scintillation counters as gamma detectors. In the present investigation these two $\beta-\gamma$ correlations are being measured with a Ge(Li) counter. A conventional fast-slow coincidence ana- . lyzer is being used with the data output being recorded onto punched pajpertape. Extensive consideration is given to both $\beta$-background and $\gamma$-background corrections.

Preliminary results are presented in Table 1.

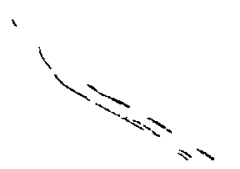

Table 1. Anisotropy coefficients of the $\beta-\gamma$ directional correlations of $\mathrm{Sb} 124$.

\begin{tabular}{|c|c|c|}
\hline$\beta$ - energy & \multicolumn{2}{|c|}{$\mathrm{A}_{22}(\overline{\mathrm{W}})$} \\
\hline $1.100-1.200(\overline{\mathrm{W}}=2.81)$ & $0.1854 \pm 0.0185$ & $-0.0514 \pm 0.0242$ \\
$1.200-1.300(\overline{\mathrm{W}}=3.04)$ & $0.2835 \pm 0.0508$ & $-0.0484 \pm 0.0229$ \\
$1.300-1.400(\overline{\mathrm{W}}=3.26)$ & $0.2979 \pm 0.0469$ & $-0.0351 \pm 0.0243$ \\
$1.400-1.600(\overline{\mathrm{W}}=3.60)$ & $0.3661 \pm 0.1350$ & $-0.1084 \pm 0.0639$ \\
\hline
\end{tabular}

The analysis of the data is in progress. 
L. Beta-Gamma Directional Correlation of K-Forbidden B-Transitions

S. Cipolla, Z. W. Grabowski, and R. M. Steffen

The $\beta-\gamma$ directional correlation of the K-Forbidden $(\Delta K=3)$ 1. $745 \mathrm{MeV} \beta$-transition with the $0.087 \mathrm{MeV} \gamma$-transition in the decay of $\mathrm{Tb}^{160}$ is under re-investigation. Some of the improved apparatus and techniques are a Ge( $(i)$ gamma detector replacing the NaI detector, a compact lucite vacuum chamber replacing the one of brass, a vacuumevaporated source instead of a drop evaporated one, and complete computer analysis of data. Also, concurrent with the $\beta-\gamma$ directional correlation measurement, the directional correlation of the $0.870 \mathrm{MeV}-0.087$ $\mathrm{MeV}$ gamma-gamma cascade is observed in order to evaluate the attenuation of the $\beta-\gamma$ directional correlation in the $0.087 \mathrm{MeV}$ excited state of Dy ${ }^{160}$. Metallic $\mathrm{Tb}$ and $\mathrm{TbCl} \ell_{3}$ sources are used alternately. A Ge(Li) $\mathrm{NaI}(\mathrm{T} \ell$ ) coincidence counter arrangement is used for the $\gamma-\gamma$ directional correlation measurements.

The analysis of the $\beta-\gamma$ directional correlation data involves several corrections. One is due to the interfering (1-3) cascade of the 1. 155 $\mathrm{MeV} \beta$ and the $0.197 \mathrm{MeV} \gamma$ transition. Furthermore, an interference arises from Compton scattering of the $0.197 \mathrm{MeV}$ gamma rays which peaks at $0.088 \mathrm{MeV}$. Another correction stems from the $\gamma$-background in the $\beta$ detector in coincidence with the $0.087 \mathrm{MeV}$ transition. Lastly, there is a possibility of $\beta$-bremsstrahlung under the $0.087 \mathrm{MeV}$ peak being in coincidence with the $1.75 \mathrm{MeV} \beta$-transitions. The results obtained so far are listed in Table 1. 
Table 1. $\quad \beta-\gamma$ Correlation Data for $\mathrm{Tb}^{160}$

\begin{tabular}{|c|c|}
\hline $\begin{array}{c}\text { Beta Energy Range } \\
\text { (MeV) }\end{array}$ & $A_{22}(\mathrm{~W})$ \\
\hline $1.150-1.300$ & $-0.1132 \pm 0.0017$ \\
$1.300-1.450$ & $-0.1123 \pm 0.0009$ \\
$1.450-1.600$ & $-0.1109 \pm 0.0013$ \\
$1.600-1.750$ & $-0.1109 \pm 0.0023$ \\
\hline
\end{tabular}

If only the second rank tensor type matrix element $\left\langle B_{i j}\right\rangle$ contributed to the $1.745 \mathrm{MeV} \beta$ transition of $\mathrm{Tb}^{160}$, as one might expect from the $K$-forbiddeners $\Delta K=3$, the anisotropy coefficient of the $3^{-}(\beta) 2^{+}(\gamma) 0^{+}$ directional correlation would be given by

$$
A_{22}(W)=-\frac{2}{7} \frac{p^{2}}{p^{2}+\left(W_{0}-W\right)^{2}}
$$

with $A_{22}\left(W_{0}\right)=-0.283$ at the maximum energy of the $\beta$-spectrum. The data in Table 1 clearly indicate that this is not the case. Hence, the vector type matrix elements must contribute significantly to the $\Delta K=3$ first-forbidden $\beta$-transition in $\mathrm{Tb}^{160}$.

A more detailed analysis of the data is in progress. 
M. Gamma-Gamma Directional Correlation Measurements with a Ge (Li) - NaI (Tl) Correlation Equipment

S. Cipolla and R. M. Steffen

Gamma-gamma directional correlation measurements with a Ge(Li)$\mathrm{NaI}(\mathrm{T} l)$ coincidence analyzer were performed on cascades in the decay of $\mathrm{Eu}^{152}$ and $\mathrm{Ta}^{182}$.

Of interest in the Eu ${ }^{152}$ decay was the directional correlation of the $868-245 \mathrm{keV}$ cascade. Interfering correlations due to background connected with each transition were also measured. Once the results have been thus corrected, the mixing ratio of the $868 \mathrm{keV}$ transition can be determined.

Several cascades were measured in the decay of $\mathrm{Ta}{ }^{182}$. Calcíliations to correct for interfering correlations are yet to be completed.

The results obtained so far are listed in Table 1 and Table 2.

Table 1. $\quad \gamma-\gamma$ Directional Correlations in the Decay of $\mathrm{Eu}^{152}$ and $\mathrm{Ta}^{182}$

\begin{tabular}{|c|c|c|c|}
\hline Isotope & Cascade (keV) & $A_{22}$ & $A_{44}$ \\
\hline \multirow{2}{*}{ Eu $^{152}-\mathrm{Sm}^{152}$} & $868-245$ & $0.1395 \pm 0.0080$ & $-0.0880 \pm 0.0165$ \\
& $1409-122$ & $0.232 \pm 0.004$ & \\
& $1210-245$ & $-0.1439 \pm 0.0203$ & $0.0236 \pm 0.0336$ \\
& $1210-122$ & $0.0370 \pm 0.0066$ & $-0.0191 \pm 0.009$ \\
$\mathrm{Ta}^{182}-\mathrm{W}^{182}$ & $67-1222$ & $+0.1554 \pm 0.0036$ & $+0.0055 \pm 0.0079$ \\
& $67-1122$ & $+0.0120 \pm 0.0087$ & $-0.0026 \pm 0.0130$ \\
& $1122-100$ & $-0.0389 \pm 0.0064$ & $+0.1066 \pm 0.0098$ \\
& $1232-100$ & $0.0196 \pm 0.0041$ & $0.0378 \pm 0.0062$ \\
& $152-1122$ & $-0.0863 \pm 0.0299$ & $-0.0430 \pm 0.0090$ \\
& $152-1222$ & $-0.0260 \pm 0.0060$ & $-0.0430 \pm 0.0090$ \\
& $222-1232$ & $-0.0179 \pm 0.0106$ & $-0.0130 \pm 0.0016$ \\
\hline
\end{tabular}


Table 2

Mixing Ratios of Some $\gamma$-Transitions in the Decay of $\mathrm{Eu}^{152}$ and $\mathrm{Ta}^{182}$

\begin{tabular}{|c|c|c|}
\hline Isotope & Transition (MeV) & Mixing Ratio $\delta$ \\
\hline $\mathrm{Eu}^{152}-\mathrm{Sm}^{152}$ & 1.409 & $-0.030 \pm 0.005$ \\
$\mathrm{Ta}^{182}-W^{182}$ & 0.067 & $-0.13 \pm 0.01$ \\
& 1.122 & $+7.35+3.53$ \\
& & 0.44 \\
\hline
\end{tabular}

N. Gamma-Gamma Directional Coŕrelation Measurements with a Ge(Li) - Ge(Li) Correlation Equipment

A. J. Becker and R. M. Steffen

A program of precise $\gamma-\gamma$ directional correlation measurements. using two Ge(Li) detectors has been started. The good energy resolution of $\mathrm{Ge}(\mathrm{Li})$ detectors makes it possible to study directional correlations in very complicated decays with a large number of gamma transitions with some degree of confidence. Many directional correlations which have been measured before with $\mathrm{NaI}(\mathrm{T} l)$ detectors are inaccurate due to the many corrections which must be applied because of the poor energy resolution of the $\mathrm{NaI}(\mathrm{T} \ell)$ spectrometers. In particular many $\mathrm{M} 1-\mathrm{E} 2$ mixing ratios, extracted from NaI-directional correlation measurements are subject to large errors and are in some cases incorrect.

The directional correlations of the gamma rays of $\mathrm{Tm}^{169}, \mathrm{Pt}^{192}$, $\mathrm{Pt}^{194}, \mathrm{~W}^{182}, \mathrm{Dy}^{160}, \mathrm{Sm}^{152}, \mathrm{Gd}^{152}$ and $\mathrm{Gd}^{154}$ are being studied at present. 
Exact knowledge of the multipole properties of the gamma transitions in the $K=\frac{1}{2}$ ground-state rotational band of $\mathrm{Tm}^{169}$ is of particular interest. Using two Ge(Li) gamma spectrometers with a two-dimensional multichannel analyzer, the directional correlations of the $\mathrm{Tm}^{169}$ gamma-rays were measured. Results for the directional correlation coefficients of the various cascades are: (I) $177 \mathrm{keV}-130 \mathrm{keV}$ cascade: $A_{22}=0.253 \pm 0.008, A_{44}=$ $0.036 \pm 0.010$; (II) $198 \mathrm{keV}-110 \mathrm{keV}$ cascade: $A_{22}=0.338 \pm 0.007, A_{44}=$ $0.018 \pm 0.011$. Correlation coefficients (I) are in fair agreement with previous, scintillation counter measurements, whereas coefficients (II) are somewhat larger and indicate a mixing ratio for the $198 \mathrm{keV}$ transition of $\delta(198)=\langle 5 / 2\|E 2\| 7 / 2\rangle /\langle 5 / 2\|M 1\| 7 / 2\rangle=0.30 \pm 0.03$. In order to determine the mixing ratio $\delta(20.8)$ of the $20.8 \mathrm{keV}$ radiation, the $177 \mathrm{keV}-110 \mathrm{keV}$ (1-3) directional correlation was found: $A_{22}=-0.314 \pm 0.010, A_{44}=$ $0.036 \pm 0.010$, and the mixing ratio $\delta^{2}\left(\gamma_{2}\right)$ was extracted from the crossover factors $U_{k k}$ in the expression for the (1-3) directional correlation coefficient, $A_{k k}\left(\gamma_{1}-\gamma_{3}\right)=A_{k}\left(\gamma_{1}\right) U_{k k}\left(\gamma_{2}\right) A_{k}\left(\gamma_{3}\right)$, yielding the value $\delta^{2}(20.8 \mathrm{keV})=0.00 \pm 0.05$.

O. Mixing Ratio of Transitions in the Decay of $\operatorname{Ir}^{192}$

Z. W. Grabowski

Mixing ratios of the $308 \mathrm{keV}$ and $604 \mathrm{keV}^{\text {transitions in } \mathrm{Pt}}{ }^{192}$ and $485 \mathrm{keV}$ transition in $\mathrm{Os}^{192}$ were measured with an automatic $\gamma-\gamma$ directional correlation apparatus equipped with a Ge(Li) detector. The source was prepared by irradiating spectroscopically pure iridium powder in the Argonne National Laboratory reactor. After Ir ${ }^{194}$ had decayed the irradiated 
Ir powder was dissolved in aqua regia and a diluted solution was used for the measurement. Data were collected at seven different angles and the correlation coefficients were calculated by fitting the experimental data to the directional correlation function in the form:

$$
W(\theta)=\sum A_{k k} P_{k}(\cos \theta)
$$

The mixing parameter $\delta$ is defined as the ratio of the emission matrix elements

$$
\delta=\frac{\langle f|L+1| i\rangle}{\langle f|L| i\rangle},
$$

and $\delta^{2}$ is the intensity ratio of the two multipoles.

The results of the measurement, corrected for the finite solid angle and contribution from the coincident background, are given in Table 1.

Table 1

\begin{tabular}{|c|c|c|c|c|}
\hline Cascade & Correlation coefficients & $\delta$ & $\begin{array}{l}\text { Level } \\
\text { (keV) }\end{array}$ & $\begin{array}{c}\text { Spin } \\
\text { Assignmert }\end{array}$ \\
\hline $308 y-612 y$ & $\begin{array}{l}A_{22}=-0.093 \pm 0.007 \\
A_{44}=-0.057 \pm 0.012\end{array}$ & -7.5 & 921 & $3^{+}$ \\
\hline $604 \gamma-316 \gamma$ & $\begin{array}{l}A_{22}=-0.492 \pm 0.015 \\
A_{44}=-0.051 \pm 0.020\end{array}$ & $\begin{array}{l}+0.75 \text { or } \\
+2.0\end{array}$ & 921 & \\
\hline $485 \gamma-316 \gamma$ & $\begin{array}{l}A_{22}=-0.274 \pm 0.011 \\
A_{44}=-0.073 \pm 0.015\end{array}$ & $\begin{array}{r}+9.5 \\
:\end{array}$ & 690 & $3^{+}$ \\
\hline
\end{tabular}

Directional Correlation Coefficients and Mixing Ratios in the Decay of Ir 192

The large mixing ratio $(\delta=-7.5)$ for the $308 \mathrm{keV}$ transition in $\mathrm{Pt}^{192}$ agrees very well with conversion coefficient ${ }^{1)}$ and $K / L$ ratio measurements 2,3). In the case of the $604 \mathrm{keV}$ transition in $\mathrm{Pt}^{192}$ the larger $\delta$ value 
$(\delta=+2.0)$ is preferred by the linear polarization measurement $\dot{4})$, which give $\delta=+1.9$. The $\delta=9.5$ for the $485 \mathrm{keV}^{\mathrm{transition}}$ in $\mathrm{os}^{192}$ agrees very well with the conversion coefficient for this transition as reported in ref. 5 .

Large mixing ratios for $308 \mathrm{keV}, 604 \mathrm{keV}$ and $485 \mathrm{keV}$ transitions indicate the collective character of the $921 \mathrm{keV}$ excited state in $\mathrm{Pt}^{192}$ and. the $690 \mathrm{keV}$ level in Os $^{192}$.

1. S. Hultberg, D. J. Horen, and J. M. Hollander, Nucl. Phys. 28, 472 (1961)

2. L. Marinkov et al., Bull Inst. Nuclear Sci. Boris Kidrich (Belgrade) 10, 7 (1960)

3. W. F. Frey, J. H. Hamilton and S. Hultberg, Ark. Fysik 21, 383 (1962)

4. W. D. Hamilton, private communication (1968)

5. L. G. Marinkov and H. T. Zupaucic, Bull. Boris Kidrich Inst. Nucl. Sci.' 13,7 (1962)

P. The g-Factors of $2^{+}$Vibrational States in Pt 192

\section{Z. W. Grabowski}

The directional correlation method was used for measuring the $\mathrm{g}$ factor of the first $(316 \mathrm{keV}) 2^{+}$excited state in $\mathrm{Pt}^{192}$. This method is based on the observation of rotation of the time-integrated correlation pattern of two cascading $\gamma$-rays in an external magnetic field ${ }^{1)}$. Due to the short halflife $\left(\sim 10^{-11} \mathrm{sec}\right)$ of this state the hyperfine magnetic field in iron was used to produce the rotation. Two sources were prepared by melting spectroscopically pure iridium and iron powder into a button using electron bombard- . ment. They differ by the iridium concentration which was $2 \%$ (by weight) in 
'the first and $4 \%$ in the second source. The diameter of each button was $\sim$ lmm. Subsequently the samples were irradiated in the Argonne National Laboratory Reactor at a flux of $5 \times 10^{12}$ neutrons $/ \mathrm{cm}^{2} \mathrm{sec}$ for two hours. The sources were not annealed after irradiation, and the measurements were started about one week after irradiation to allow for the 19 hours activity due to $\operatorname{Ir}^{194}$ to decay.

The coincidences between the $468 \mathrm{keV}$ gamma and the $316 \mathrm{keV}$ gamma ray were recorded at seven different angles for both directions of the magnetic field. The $\omega \tau$ (and $A_{22}$ ) was calculated from the experimental data by fitting the

$$
W(\theta, \pm B)=\sum_{k=0}^{2} G_{k k} A_{k k} P_{k}[\cos (\theta-\omega \tau)]
$$

function to the measured values. The results of the measurements are listed in Table 1.

Table 1. Rotation of Directional Correlation

\begin{tabular}{|c|c|c|c|c|}
\hline Source & Field direction & $\omega \tau$ & $\overline{\omega \tau}$ & $\mathrm{A}_{22}$ \\
\hline \multirow{2}{*}{$2 \% \mathrm{Ir}$} & up & $-0.083 \pm 0.028$ & $0.057 \pm 0.014$ & $0.099 \pm 0.006$ \\
& down & $0.048 \pm 0.015$ & & $0.009 \pm 0.004$ \\
$4 \% \mathrm{Ir}$ & up & $-0.081 \pm 0.010$ & $0.080 \pm 0.008$ & $0.100 \pm 0.002$ \\
& down & $0.078 \pm 0.019$ & & $0.098 \pm 0.003$ \\
\hline
\end{tabular}

Values for $\overline{\omega \tau}$ listed in the table are weighted averages of each set of measurements with magnetic field up and down.

The weighted average of the present measurements listed in the table

$$
\omega \tau=0.073 \pm 0.007
$$


This value gives the $\mathrm{g}$-factor for the $316 \mathrm{keV} 2^{+}$level in $\mathrm{Pt}^{192}$

$$
g=0.23 \pm 0.03 \text {. }
$$

For calculation of this value the magnetic field, $H_{\text {int }}=1320 \pm 80 \mathrm{kG}$, w,as used and the mean life for the $316 \mathrm{keV}$ state was taken to be $T=(5.1 \pm 0.4) \mathrm{x}$ $10^{-11} \mathrm{sec}$, as reported in ref. 2 .

The results of the other authors

$$
\begin{aligned}
& \omega \tau=0.063 \pm 0.0093) \\
& \omega \tau=0.080 \pm 0.005 \text { 4) and }
\end{aligned}
$$

are in good agreement with our measurements. The value for the g-factor is significantly lower than the theoretical value, $g_{\text {th }}=0.334$, obtained from the model proposed by Greiner ${ }^{5}$ ). The strength of the pairing forces for protons and neutrons used for calculating $g_{\text {th }}$ was taken from $x$ ef. 6 .

1. H. Frauenfelder and R. M. Steffen in " $\alpha, \beta$ and $\gamma$-Ray Spectroscopy", edited by K. Siegbahn, North-Holland Publishing Co. (1965).

2. A. Schwarzschild, Phys. Rev. 141, 1206 (1966)

3. L. Keszthelyi, I. Berkes, I. Dezsi and L. Pocs, Nucl. Phys. 71,662 (1965)

4. Y.K. Agarwal, C. V.K. Baba ànd S. K. Bhattacherjee, Nucl. Phys. 79, 437 (1965)

5. W. Greiver, Nucl. Phys. 80, 417 (1966)

6. E. R. Marshallek and J. O. Rasmussen, Nucl. Phys. 43, 438 (1963) 
Q. The Magnetic Hyperfine Field at Hf Nuclei in an Iron Lattice

A. J. Becker and R. M. Steffen

The method of spin precession as observed in a time-integrated directional correlation measurement has been used to determine the induced magnetic hyperfine field $\mathrm{H}_{\text {int }}$ acting at $\mathrm{Hf}$ nuclei embedded in an iron lattice. The Larmor precession angle of the $9 / 2^{+}(208 \mathrm{keV}) 9 / 2^{-}$ $(113 \mathrm{keV})^{7} / 2^{-}$gamma-gamma directional correlation in $\mathrm{Hf}^{177}$ using a source of $\mathrm{Lu}^{177}$ implanted in an iron foil was measured and from the known $\mathrm{g}$-factor of the $113 \mathrm{keV}$ state of $\mathrm{Hf}^{177}$ the magnitude and sign of $H_{\text {int }}$ was extracted as, $H_{\text {int }}=-(300 \pm 60) \mathrm{k}$ Oersted. The influence of quadrupole interactions could not be neglected and appropriate corrections were applied to the measurements.

The value and sign of $\mathrm{H}_{\text {int }}$ for $\mathrm{Hf}$ in iron agrees reasonably well with the empirical systematic trend shown by $\mathrm{H}_{\text {int }}$ for the $5 \mathrm{~d}$ transition elements in an iron host.

The results of this investigation have been reported in the Proceedings of the International Conference on Hyperfine Interactions Detected by Nuclear Radiations, Asilomar (Calif.) August 25-30, 1967 (North-Holland Publishing Co., Amsterdam, 1968). 
R. Alpha-Gamma Directional Correlation Measurement in the Decay of $\mathrm{Th}^{230}$

Z. W. Grabowski

In order to investigate in detail the influence of extranuclear effects and alpha-recoil on the directional correlation, the $4.61 \mathrm{MeV} \alpha-68 \mathrm{KeV} \gamma$ cascade in $\mathrm{Th}^{230}$ decay has been measured. The results for the attenuation coefficients in the directional correlation function

$$
W(\theta)=1+G_{22} A_{22} P_{2}(\cos \theta)+G_{44} A_{44} P_{4}(\cos \theta)
$$

are

$$
\begin{aligned}
& \mathrm{G}_{22}=0.38 \pm 0.02 \\
& \mathrm{G}_{44}=0.49 \pm 0.03
\end{aligned}
$$

This is in agreement with the result obtained by Temmer and Wyckoff showing higher attenuation than reported by Vallados et al. 2). The experimental results $G_{4}>G_{2}$ proves the presence of quadrupole interaction.

1) G. M. Temmer and J. M. Wyckoff, Phys. Rev. 92, 913 (1953)

2) G. Vallados et al., J. Phys. Rad. 16, 125 (1955)

S. Hyperfine Field of $\mathrm{Rh}^{103}$ in Iron and the $\mathrm{Rh}^{103}-\mathrm{Rh}^{100 \mathrm{~m}}$ Hyperfine Anomaly in Iron

W. C. King and R. P. Scharenberg

It is of interest to measure accurately the magnetic hyperfine fields existing at the nuclei of impurity atoms imbedded in ferromagnetic host 
lattices, both from the point of view of testing the related magnetism theories, and for the use that can often be made of these generally quite large fields in various experiments. (See Sections $Q$ and $P$ of this chapter). From the latter point of view it is usually desirable that the temperature dependent fields be known accurately at room temperature, although for sensitivity reasons, it is often easier to measure the hyperfine fields at reduced temperatures.

We have measured the hyperfine field of $\mathrm{Rh}^{103}$ in ir on at $305.5^{\circ} \mathrm{K}$ in zero external field by steady-state nuclear magnetic resonance methods. A frequency modulated "Robinson oscillator" was employed. Very low RF levels were used with a modulation frequency of $500 \mathrm{cps}$. The resonance signal was detected by means of a lock-in amplifier operating at the second harmonic of the modulation frequency. A 3 second RC filter time constant was used on the lock-in amplifier and the output was fed to a digital memory where successive sweeps through the resonance were coherently added for a further increase in the signal to noise ratio. Figure 1 shows the resultant trace after approximately 150 sweeps through the resonance. The resonance curve has a FWHM of approximately $125 \mathrm{Kc}$ and a center frequency of $69.77 \pm \mathrm{x} \mathrm{Mc}$ at $\mathrm{T}=305.5^{\circ} \mathrm{K}$.

The' evaluation of the data is in progress. 


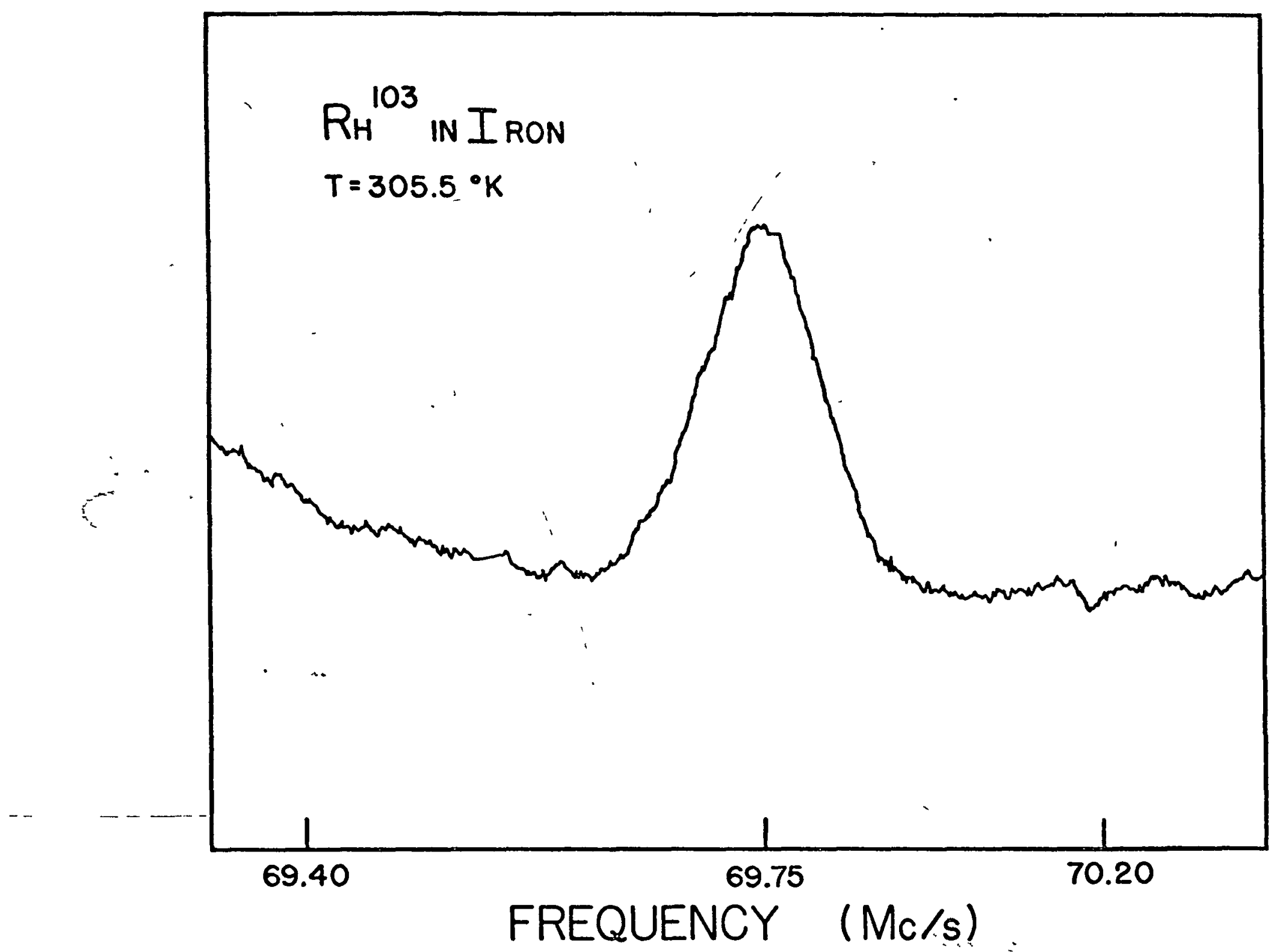

Fig. 1. $\mathrm{Rh}^{103}$ resonance in iron at $\mathrm{T}=305.5^{\circ} \mathrm{K}$ and with zero external field. Center frequency is $(69.75 \pm .02) \mathrm{Mc} / \mathrm{s}$ and the FWHM is approximately $125 \mathrm{Kc} / \mathrm{s}$. 
T. Angular Correlation Handbook

\author{
R. M. Steffen
}

A collection of formulae, tables and graphs useful to research groups engaged in experimental work on angular correlations was prepared. No derivations of formulae and few references to original work are given. It was not intended to write a review paper on angular correlation, but rather to make available to interested experimentalists, graphs, tables and formulae that are in constant use in the Purdue Nuclear Physics Laboratory.

Following a request by Dr. K. Way, editor of Nuclear Data, the Angular Correlation Handbook is now being prepared for publication in Nuclear Data, Section A. 


\section{GENERAL NUCLEAR SPECTROSCOPY}

\section{A. Short Lifetime Measurements}

\section{P. C. Simms and H. Kuhlman}

A new experimental technique has been developed for measuring the lifetime of excited states. With this system, the centroid-shift method can be applied to many isotopes with a greater accuracy than was previously possible. Two plastic scintillators are used to detect the particles which indicate the formation and decay of the excited state. In the simplest experimental arrangement, one of these plastic scintillators is surrounded by a Na I detector. (The plastic detector is placed in a hole which is drilled through the $\mathrm{Na} I$ detector perpendicular to its symmetry axis. The plastic and $\mathrm{Na} I$ detectors are viewed by separate P. M. tubes.)

As an example of the method, consider a state where a 0.7 and 1.0 Mev gamma ray are emitted when the state is formed and then decays. Single-channel analyzers connected to the two plastic scintillators are set to record an energy loss between 400 and $450 \mathrm{keV}$. Either gamma ray can be detected in either scintillator. However, the energy of the scattered gamma ray which leaves the plastic scintillator will depend on the energy of the incident gamma ray. The scattered gamma rays are detected in the $\mathrm{Na} I$ scintillator. Thus the plastic detectors cannot distinguish between the particles, but the $\mathrm{Na} I$ detector can be used to determine which particle hit which detector.

The plastic detectors are also connected to the inputs of a time-to amplitude converter. (See electronics section). The output from the time 
converter is passed to a multichannel analyzer when a separate coincidence circuit indicates that the scattered gamma ray has been recorded in the Na I detector. A pair of single-channel analyzers connected to the $\mathrm{Na}$ I detector determines which particle hit which detector and routes the corresponding time pulse to different sections of the MCA memory. The difference between the centroids of the two time distributions in the MCA will be related to the mean life of the excited state.

There are many advantages to this method. First, the effect is twice the lifetime of the state, since both time distributions are being recorded at the same time. Any shifts in the electronics will effect both distributions in the same way and thus not effect the measurement. The technique can be applied to any state where the gamma rays can be distinguished in a $\mathrm{Na} \mathrm{I}$ detector. Co ${ }^{60}$ has been used in calibration measurements, and there is no difficulty in distinguishing between the two scattered gamma rays even though the incident gamma rays differ in energy by only $10 \%$. Of course the window settings of the plastic detector single-channel analyzers must be relatively narrow, but this is usually necessary in any arrangement to get good time resolution.

High counting rate linear amplifiers and single-channel analyzers (see electronics section) are used for the plastic detectors so that data is accumulated rapidly. The $\mathrm{Na} \mathrm{I}$ detector is shielded from direct radiation from the source so that its counting rate will not be excessive. Most measurements will require only a few days to perform.

A computer calculation has been performed to estimate the systematic error in the experiment. Since the incident particles have different energy, their spectrum shapes are not identical. The single-channel analyzers are set to minimize the energy variation, across the window. Any remaining 
systematic effect due to differences in amplitude walk is estimated by the computer. The flight time of the gamma rays, scintillation light, and photoelectrons have also been considered.

Preliminary measurements on the life-time of the first excited state of $\mathrm{Ni}^{60}$ shows that the total systematic and statistical error will be less than $10^{12} \mathrm{sec}$. for this nucleus. Measurements are also in progress on $\mathrm{Ti}^{46}$, $\mathrm{Cd}^{114}, \mathrm{Pt}^{192}$. It is expected that an accuracy of better than $10^{-12}$ seconds can also be obtained for these measurements.

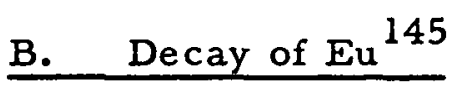

John C. Hill

The decay scheme of $\mathrm{Eu}^{145}$ was studied by means of the techniques of beta and gamma spectroscopy. The half-life was measured to be $5.93 \pm 0.1$ days. Thirty-three gamma transitions were identified by means of NaI and germanium gamma detectors and $180^{\circ}$ magnetic spectrometers. Gammagamma coincidences were performed using both $\mathrm{NaI}$ and germanium detectors. Most of the transitions were incorporated into the decay scheme in a consistent manner. The multipolarity of several gamma transitions was determined from measurement of conversion coefficients, and a positron spectrum containing two branches was observed. The level scheme of $\mathrm{Sm}^{145}$ contains a first excited state at $0.895 \mathrm{MeV}$ and a second excited state at $1.437 \mathrm{MeV}$. Above 1.5 $\mathrm{MeV}$, the level density greatly increases. Levels with both even and odd parities were observed, and the energy between' the ground states of $\mathrm{Eu}^{145}$ and $\mathrm{Sm}^{145}$ was determined to be about 2. $75 \mathrm{MeV}$. Systematics for the first excited states of nuclei with even proton number and a neutron number near 82 are presented.

The results of this investigation have been published in The Physical Review, 153, 1312 (1967). 


\section{INSTRUMENTATION}

\section{A. The Purdue Broad-Range Magnetic Spectrograph}

E. Bleuler, D. J. Tendam, J. W. Hemsky, and C. L. Wiley

The Purdue broad-range magnetic spectrograph is a modified version of the high-resolution particle spectrographs designed and built at M. I. T. and at the University of Notre Dame, The Purdue instrument has three distinguishing features. (1) It has a horizontal rather than the conventional vertical axis of rotation for the spectrograph magnet. The advantages of this construction are twofold. First, the energy spread in the reaction products is less for scattering in a vertical plane than for scattering in the horizontal plane. Second, the design of the scattering chamber is simpler for this incident-beam geometry. The chief disadvantage of the horizontal-axis configuration is that the gravitational torque on the spectrograph magnet is different for each angular position of the magnet. This situation causes small shifts in the incident-beam direction W (and hence in the incident-beam energy) and in the nominal scattering angle, as the magnet is rotated to accept particles having different scattering angles. (2) The Purdue spectrograph is designed to have second-order focusing at three positions along the focal surface. This focusing property has been achieved by machining the contour of the exit magnetic-field boundary to consist of three continuous arc segments, each having a different radius of curvature. There appears to be second order focusing at the positions near $s=9 \mathrm{~cm}, s=36 \mathrm{~cm}$, and $s=53 \mathrm{~cm}$. Due to the complicated shape of the exit magnetic-field boundary, no single, closed, analytic expression could be derived to describe the focal surface. For this reason, 
the energy calibration equation for the focal surface consists of several piecewise-continuous functions, each of which is valid over a specified region of the focal surface. These functions were least-squares fitted to the experimental calibration data under the constraint that each function and its first derivative be continuous with the function and its first derivative, respectively, in the adjacent region of the focal surface. To facilitate their use, the focal-surface calibration equations have been used to generate tables of the calibration information. (3) The design of the Purdue spectrograph incorporates focusing effects in the direction perpendicular to the median plane; these focusing effects enhance the solid angle of the spectrograph by a factor of approximately 1.8 .

The field coils of the spectrograph magnet can generate magneticfield strengths up to 17 Kgauss in the 1 -inch magnet gap through which the charged reaction products are deflected to the $75-\mathrm{cm}$-long calibrated portion of the focal surface where they are detected in an array of nuclear-track emulsions. For magnetic-field streng ths below 9.5 Kgauss, alpha particlés from 210 po were used to perform the energy calibration of the spectrograph focal surface. The validity of the resulting low-field energy-calibration equation for the spectrograph was investigated at higher magnetic-field strengths; and, within the limitations imposed by the fixed energy of the deuteron beam from the Purdue cyclotron, the low-field energy-calibration equation appears to be accurate to within $0.1 \%$ for magnetic-field strength below 13. 75 Kgauss.

The average (energy) resolving power of the spectrograph focal surface is $1.2 \cdot 10^{3}$ for a $1^{\circ}$ half-angle of acceptance and is $0.87 \cdot 10^{3}$ for a $3^{\circ}$ half-angle of acceptance. The solid angle of the spectrograph in a $1 / 2$-inch by $5 / 8$-inch entrance-mask and 0.418 -inch exit-mask configuration varies 
smoothly from $0.43 \mathrm{~m} \mathrm{sr}$ at $\mathrm{s}=0$ to $0.30 \mathrm{~m} \mathrm{sr}$ at $\mathrm{s}=75 \mathrm{~cm}$.

By using the spectrograph low-field energy calibration as a standard, an energy-calibration equation for the incident-beam-energy analyzing magnet has been obtained; the accuracy of the analyzing-magnet energy-calib'iation equation is $0.1 \%$.

The calibration of the Purdue broad-range magnetic spectrograph is now completed, and the results are available in a formal report. ${ }^{1)}$ The instrument has been utilized successfully in several experiments which have required very high $(<1 / 2 \%)$ resolution.

1. E. Bleuler, D. J. Tendam, J. W. Hemsky, and C. L. Wiley, "The Calibration of the Purdue Broad-Range, Magnetic Spectrograph and the Incident-Beam-Energy Analyzing Magnet" (COO-1420-123; Purdue Research Foundation, Lafayette, Indiana, unpublished).

\section{B. Construction of $18^{\prime \prime}$ Scattering Chamber}

G. Schilling, R. P. Scharenberg, and J. W. Tippie

An 18 inch scattering chamber designed for particle gamma-ray studies has been designed and built.

The chamber walls are composed of a $7 "$ high and 18" diameter vacuum collar obtained from the Materials Research Corporation (MRC). The bottom plate includes a coaxial drive system for two independently controllable counter arms and target changing mechanism. The advantage of this design is that the top plate is completely available for gamma ray detectors. The scattering chamber mechanisms are Selsyn controlled and can be - 
actuated from remote locations. An auxilliary 4 " diffusion vacuum system has been installed on the chamber, and is capable of maintaining the pressure below $10^{-6}$ Torr.

The chamber has been instrumented for the Coulomb excitation experiments described elsewhere in this report. This involves the installation of a 10" diameter, 4" thick $\mathrm{NaI}(\mathrm{T} l)$ crystal above l"from the target position. A $1 / 8^{\prime \prime}$ thick Be window serves to minimize absorption of the gamma rays. The chamber and $\mathrm{NaI}(\mathrm{T} l)$ crystal are surrounded by a 2 " $\mathrm{Pb}$ shield to reduce room background.

The chamber, collimators, shield, vacuum system et al. has been in use at the Argonne National Laboratory for the last two years.

C. Electronic Instrument Development

P. C. Simms

A number of general purpose electronic instruments have been developed. Instruments with these capabilities are not available commercially. They are packaged in the standard AEC modules so that they can conveniently be used with commercial instruments. All circuits are built on printed cir cuit boards using low cost plastic transistors and integrated circuits. Automatic Data Recording System. An automatic data recording system has been developed for the general use in Purdue Nuclear Physics Laboratory. The system is very flexible and reliable, and still it can be built for a small fraction of the cost of commercial equipment. The system is composed of decade counting cards, input cards, and control cards which permit the system to print out data on a Teletype $\mathrm{TC}_{4}-33$ page printer and paper tape punch. Any 
counting configuration can be set up by inserting the desired number of cards in a prewired card file. Each card file will accept 19 cards, and any number of card files can be operated by one master control unit. Any combination of input and count cards may be used. For example, with a single file, one could have 9 one-digit scalers $(9$ count cards and 9 input cards), or a single 18-digit scaler could be obtained with one input card and 18 count cards. No wiring changes are required to change the counting configuration. The master control unit transfers the data from each card to the printer-punch in a serial fashion. A printout circuit on the input card can be used to write identification numbers or to generate functions (space, tabulate, etc. ) for the printer.

There is a test pulse generator in the master control unit. When this test generator is turned on, all regular inputs are turned off automatically, and the same test pulse is presented to all input boards in the system. Therefore, in the test mode, all scalers should count at the same rate. The complete system can be tested in a few seconds without changing any connections.

Twin Single Channel Analyzer. This module contains two single channel analyzers which take their reference voltage from a common source. The discriminators are adjusted so that equal settings of the front panel dials will give equal window widths. This instrument is used with a time-toamplitude converter to measure true and accidental coincidences at the same time.

High Voltage Distribution Module. This instrument contains high voltage. emitter followers so that several photomultiplier tubes can be operated from one high voltage supply. The emitter followers can be controlled by potentio- 
meters mounted on the front panel or from external, feedback voltage stabilizers. The module also contains a voltage monitoring system which can detect a change in output voltages as small as 1 volt. Double Delay Line Clipped Linear Amplifier. The most important feature of this amplifier is that delay lines from 100 nanoseconds to 5 microseconds may be used. The delay lines are easy to change in plug-in sockets. Thus the amplifier can be used for extreme high counting rate with short delay lines, or it can be used for high resolution experiments with long delay lines and integrations. The gain can be varied from 700 to 20 . With a $1 \mu \mathrm{sec}$ integration and full gain the noise at the output is less than $5 \mathrm{mv}$ RMS. The noise goes down linearly with gain. With $1 \mu$ sec delay lines, the amplifier recovers from a 100 times overload pulse in less than $5 \mu \mathrm{sec}$. The area balance of the bipolar pulse and the recovery to baseline can be adjusted for optimum performance.

Single Channel Analyzer. The most important feature of the SCA is that it can process the $100 \mathrm{n}$ sec DDL amplifier pulses at counting rates greater than $1 \mathrm{MHz}$. Even with these narrow input pulses the definition of the window edges is less than $10 \mathrm{mv}$. When $750 \mathrm{n} \mathrm{sec}$ DDL pulses are used, the definition of the window edge is approximately $3 \mathrm{mv}$. The output pulse from the SCA can be timed from a zero-crossing pickoff or from low level leading edge discriminator. The leading edge and zero-crossing levels are independently adjustable. Since the window edges are so sharp, a very narrow window and leading edge timing can be used to give timing resolution of approximately $5 \mathrm{n} \mathrm{sec}$ with high resolution detectors. PM Tube Head and Preamplifier. This instrument is intended for high counting rate experiments. Transistor voltage regulators are used for the

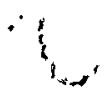


last four high current dynodes. This arrangement reduces the P. M. gain shift at high counting rates by an order of magnitude. A linear preamp is included which has a dynamic range of greater than 10 volts and a rise time of less than $10 \mathrm{nsec}$. A fast amplifier is also included for timing pulses. Multicoincidence Module. This is a general purpose instrument for moderate speed coincidence work. Each of the 4 input channels has a delay which is variable from $50 \mathrm{n} \mathrm{sec}$ to 1 millisecond. The inputs can be connected to either of two coincident circuits by movable plugs. Many arrangements are possible. For example the instrument can be connected as a quadruple coincidence, two double coincidence, 2 triple coincidence with two channel common, etc. The resolving time is adjustable from $10 \mathrm{n}$ sec to 1 millisecond.

Time-to-Amplitude Converter. This instrument is designed for very fast resolving time work. The time range of the instrument can be adjusted from $20 \mathrm{n} \mathrm{sec}$ to $1 \mu \mathrm{sec}$. The time resolution is less than $0.1 \%$ of the full scale time. The integral linearity and stability of the instrument is less than $0.1 \%$. The input pulses may be positive or negative, and they do not have to be preshaped. The input sensitivity is $50 \mathrm{mv}$. No delay lines are used in the instrument. The start input can accept counting rates larger than $1 \mathrm{MHz}$, and the stop input can accept counting rates above $50 \mathrm{MHz}$. There is no output pulse if a stop pulse does not occur within the selected time range following a start pulse.

Voltage and Current Monitor. This module indicates the current and voltage being taken from the AEC standard power supplies. This is considered necessary to use the power supplies efficiently and to avoid overloading the power supplies in high counting rate experiments. The module 
also contains a safety circuit which turns off all of the power supplies if one supply fails. This will prevent damage to modules which are using . the power supply system.

\section{Fabrication of Lithium-Drifted Germanium Detectors}

\section{A. J. Becker}

The need for Iithium-drifted germaniurn detectors for bigh-resolution gamma-ray spectroscopy has prompted the development of a fabrication technique and apparatus. Up to the present, all attempts have been to form planar detectors of up to $6 \mathrm{~mm}$ depletion depth, this value being a reasonable upper limit from $r$ ise-time considerations. Three crystals cut from a Sylvania horizontally-grown ingot have been successfully drifted, and one cut from a Hoboken vertically-grown ingot is in the final stages of drifting. Two of the Sylvania crystals have been fully prepared and give quite satisfactory results. The larger one, with $6 \mathrm{~cm}^{2}$ surface areai and $5.5 \mathrm{~mm}$.depletion depth, is presently being used on the $\gamma-\gamma$ directional correlation apparatus. Measurements using it with an Ortec Model 118 FET preamplifier, an Ortec Model 220 linear amplifier, and a Victoreen SCIPP 1600-channel multichannel analyzer have produced a system resolution of 2. $7 \mathrm{Kev}$ FWHM for the $\mathrm{Cs}^{137} 664 \mathrm{Kev} \gamma$-ray line.

The cylindrical shape of the Hoboken germanium ingot makes it especially suitable for use in the fabrication of open-ended coaxial detectors. Present work involves the modification of the existing apparatus and techniques to enable the manufacture of these large-volume, higher-efficiency detector 8 . 
VI. COMPUTER PROGRAMS FOR DATA ANALYSIS

A. Non-Relativistic Kinematics for Nuclear Reactions -

Two-Body Final States

B. T. Lucas

The program calculates the energy, $E_{3}$, of the "light" final-state particle for angles of emission, $\theta_{3}$, between $0^{\circ}$ and $180^{\circ}$ (except when the kinematics imposes an upper limit on $\theta_{3}$ ) with an angular increment that is specified as input information. Also calculated are the quantities : $d E_{3} / d \theta_{3}, d E_{3} / d E_{1}$ (where $E_{1}$ is the incident energy); the center-of-mass. angle; the solid-angle ratio between the laboratory and center-of-mass systems; the angle of emission of the "heavy" final-state particle and its energy $\left(\dot{\theta}_{4}\right.$ and $E_{4}$, respectively); and the quantity $d E_{4} / d \theta_{4}$. Nine values of the excitation energy of the final nucleus may be entered on one input card and run sequentially.

B. Non-Relativistic Reaction Kinematics for Nuclear Reactions Three-Body Final States

B. T. Lucas

.':

When a nuclear reaction involving three particles in the final state is observed in such a way that coincidences are required between two of the three particles emitted at fixed angles $\theta_{1}$ and $\theta_{2}$, the corresponding energies ' $E_{1}$ and $E_{2}$ will lie on a continuous curve. The program calculates this kine- 
matic locus $\left(E_{1}, E_{2}\right)$ for the coincident events corresponding to fixed pairs of angles $\left(\theta_{1}, \theta_{2}\right)$. Also calculated are the energy and angle of the third particle and the phase-space factors $N\left(E_{1}, \theta_{1}, \theta_{2}\right), N\left(E_{2}, \theta_{1}, \theta_{2}\right) 1$. Up to eight pairs of angles $\left(\theta_{1}, \theta_{2}\right)$ may be entered on one data card and run sequentially.

\section{Finite Target Thickness Calculation}

\section{B. B. Srivastava}

The program can calculate the target thickness from a knowledge of the energy lost by a heavy charged particle of known mass, charge, and initial energy in passing through it. The target may be a mixtur.e and/or compound of known relative constitution consisting of a maximum of five different elements. In the program, the total energy loss is partitioned into a number of small, equal energy-intervals, the stopping power, $d E / d x$, is assumed constant in each energy increment and an equivalent thickness (areal density) is calculated, the thickness of these slabs is totaled to give the target thickness. In the output are included: the $d E / d x$ for each constituent element and the total $\mathrm{dE} / \mathrm{dx}$ for the mixture in each of the energy intervals; the areal density corresponding to each energy interval, and the accumulated areal density up to each energy interval.

As an option the program can calculate the energy lost by a heavy charged particle of known.mass, charge, and initial energy in passing through a given slab of material of thickness. In this case, the total thickness of the slab is partitioned into a number of small equal increments. The energy loss is then calculated in a straightforward manner. The output in this mode of operation includes: the energy loss in each increment; the 
accumulated energy loss up to each increment; and the total $\mathrm{dE} / \mathrm{dx}$ appropriate to each increment for the mixture and each of the constituent elements.

\section{Program for Analysis of Angular Correlation Data}

\section{A. J. Becker}

A computer program has been written to test, analyze, and reduce the data obtained from an angular-correlation experiment. It is in Fortran IV language and can be run with minor modifications on any large-scale computer.

Upon input, the data is checked for obvious errors such as incorrect count-periods, groups of zero-counts, and incorrect angle references. Then optionally the decay constant of the data from each register can be found through a linear least-squares fit or can be entered by the experimenter so that this decay can be adequately compensated for to enable statistical testing.

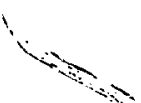

Either a chi-squared test or one limiting the standard deviation of the average can then be performed on the data for each angle between the two detectors. Highly deviant runs can thus be recognized and rejected. The accepted coincidence data is then normalized to remove the effects of a possible imprecision in the source positioning and of instrumental drifts, and the true-coincidence count-rates and their probable errors and standard deviations determined for each angle.

A non-linear least-squares fit then determines the parameters $b_{i}$ in the perturbed-angular-correlation function

$$
W(\theta)=b_{2}+b_{3} P_{2}\left(\cos \left(\theta-b_{1}\right)\right)+b_{4} P_{4}\left(\cos \left(\theta-b_{1}\right)\right)
$$


and plots the data points and the predicted curve.

In the case in which two-parameter coincidence-data arrays are accumulated in the multichannel analyzer, the program has additional functions. The array from each individual run is first checked for obvious flaws such as spurious counts. Then the position of the main peak is determined and the data adjusted linearly until this peak lies in a specific channel. Then, when the arrays for each angle are summed, the peaks all occur at the same place and the effects of any long-term gain shifts have been minimized.

Finally, after the normalized true-coincidence count array have been found for each angle, a non-linear least-squares-fit separates the peak from the background and enables the determination of the angular-correlation coefficients from these peaks alone, removing the error introduced by the backgr ound contribution.

\section{E. Program for Analysis of $\beta-\gamma$ Angular Correlation Data}

in Terms of $\beta$-Matrix Elements

H. A. Smith and P. C. Simms

Two programs have been developed for use in extracting nuclear matrix elements from beta decay experiments. One program is used to study the feasibility of beta decay experiments. , Current experimental data is used to determine which matrix elements are consistent with the data. The results are arranged in such a way that one can tell which experiments would be most useful in improving the accuracy of the results. The other program is used when the data is sufficiently accurate to give a well defined 
result. It handles the matrix element in a different way so that meaning ful limits of error are established. Both programs will accept a large variety of experimental data: beta-gamma directional correlation, betagamma circular polarization, beta spectrum, nuclear orientation, and ft measurements.

F. Program for Computation of Internal Conversion Coefficients and Directional Correlation Particle Parameters Including Higher Order Effects

This program is described in Section VII, F.

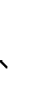




\section{THEORY}

\section{A. Large-Spin Rotational States of Deformed Nuclei}

\section{S. M. Harris}

This work was motivated by recent measurements of high-spin energy levels in even rare earth nuclei. As one reaches higher spin states in a given nucleus, the energy spacing becomes significantly smaller than that predicted by the simple $I(I+1)$ law for quantum-mechanical rotors. It seems as though the effective moment of inertia increases with spin.

We try to understand this effect by considering those terms which are fourth order in $w$, the rotational angular velocity, in the usual cranking model formulation. The results of the cranking model are rederived using fourth order perturbation theory. A two parameter formula for E vs. I is developed. Best fits for these parameters are empirically determined for several nuclei, and the resultant theoretical spectra are compared with experiment.

The results of this investigation have been published in Phy. Rev. Letters, 13. 663 (1964). 


\section{B. Higher-Order Corrections to the Cranking Model}

\section{S. M. Harris}

The results of the previous paper are extended and put on a more satisfactory theoretical basis. The energy levels of the ground-state bands of several deformed even rare earth nuclei are calculated using a scheme whereby higher order terms in $w$ are retained in the cranking model. Twoparameter and three-parameter fits are obtained to the absolute energies. The perturbation theory result is rederived using self-consistent arguments which do not depend on the quasi-adiabatic approximation. The two-parameter fits to the experimental data are comparable to the best two-parameter fits obtained by other means. Our three-parameter fits are significantly better, well within the experimental uncertainties in all but one case.

The results of this investigation have been published in the Physical Review, 138, B509 (1965). 
C. Quantum-Mechanical Model of Mössbauer Line Narrowing

Samuel M. Harris

A model for Mössbauer radiation has been developed which includes both radiation and lattice-relaxation processes. The model describes an initially excited nucleus in an excited lattice. Both radiation and relaxation phenomena are treated in lowest order, so that a quantum-mechanical solution is possible. It is found that under certain conditions, Mössbauer lines result which are narrower than the natural line, al though line broadening is the more usual effect of lattice relaxation. The maximum narrowing in this approximation never exceeds $36 \%$ of the natural width.

The results of this investigation have been published in The Physical Review, 163, 280 (1967).

D. Perturbation of Angular Correlations by Rhombic

Electric Field Gradients

E. Matthias * and R. M. Steffen

The influence of axially symmetric electric fields on angular correlations has been treated theoretically both for single crystal sources ${ }^{1)}$ and for polycrystalline (powder) sources ${ }^{2}$ ). It has been suggested earlier ${ }^{3}$ ) that higher order effects, like non-axially symmetric (rhombic) electric field gradients and/or a finite distribution of interaction frequencies will modify

*

Lawrence Radiation Laboratory, University of California, Berkeley, California.

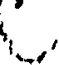


the effects of the quadrupole interaction significantly. Stimulated by some recent experimental results ${ }^{4-6}$ ), a theoretical analysis of the rhombic electric field interaction with and without the presence of applied magnetic fields was made.

$$
\text { Explicit expressions for the perturbation coefficients } G_{k_{1} k_{2}}^{N_{1} N_{2}}(t) \text { in }
$$
the directional correlation expression:

$$
\begin{gathered}
W\left(\vec{k}_{1}, \vec{k}_{2}\right)=\sum_{k_{1}, k_{2}, N_{1} N_{2}} A_{k_{1}}(1) A_{k_{2}}(2) G_{k_{1}} k_{2}(t)\left[\left(2 k_{1}+1\right)\left(2 k_{2}+1\right)\right]^{\frac{1}{2}} \\
\times \quad Y_{k_{1} N_{1}}^{*}\left(\vec{k}_{1}\right) Y_{k_{2} N_{2}}\left(\vec{k}_{2}\right)
\end{gathered}
$$

have been derived, and computer programs for their numerical calculation have been written. In view of the many independent variables (8) that occur in the expression for the perturbation coefficient general tables of these coefficients would fill many volumes. The program was run for a selected choice of input parameters that are of particular interest to experimentalists.

1) K. Alder et al., Helv. Phys. Acta. 26, 761 (1953).

2) A. Abragam and R. V. Pound, Phys. Rev. 92, 943, (1953).

3) E. Matthias, W. Schneider and R. M. Steffen, Phys. Letters 4, 4 (1963).

4) R. W. Sommerfeldt and L. Schecter, Phys. Letters 3, 5 (1962).

5) R. W. Sommerfeldt et al., Phys. Rev. 138, B 763 (1965).

6) M. Solomon et al., Phys. Letters 9, 113 (1964). 
E. Theory of Angular Distributions and Correlations of Nuclear Radiations

R. M. Steffen

A general treatise of the theory of angular distributions and correlations of nuclear radiations is being written. Full use of the formalism of statistical tensors and efficiency tensors is made and their use in computing any type of angular distribution phenomenon in nuclear physics is discussed in detail. Emission (or absorption) of radiation and their obser vation is described in terms of "linkage factors", which are simply the coefficients of expansion of the statistical tensors of the nuclear state after emission (or absorption) of radiation in terms of the statistical tensors of the initial state. A monograph on this subject is being prepared.

\section{F. Calculations of Internal Conversion Coefficients and} Particle Parameters for Finite Size Nuclei

H. C. Pauli

The major goal of this work was the computation of the conversion coefficients and particle parameters of electrons ejected from the $M$-subshells. In addition a complete computer program for calculations of the K- and L- shell particle parameters was prepared.

In these calculations the screening of the atomic electrons as well as all finite nuclear size effects were taken into account. The $M$-shell calculations were the first of its kind.

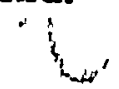


With the final program it is possible and economical to compute the internal conversion coefficient or particle parameter of any of the $K, L$, or $M$ subshells for each nuclear transition directly, avoiding the detour of interpolating from tabulated values. The next section contains an outline of the necessary computer time. Extensive check calculations for the investigation of the numerical stability and the precision of the routines for all parameter values were performed. Several variational calçulations, investigating the influence of the atomic screening assuming different screening models like the Thomas-Fermi-Dirac model or non-relativistic HartreeFock calculations were made. Some selected results of the se calculations will be published soon.

The total program consists of about 200 statements divided into 44 subroutines, and the main program, and is thus too voluminous to be described here in detail. We shall restrict ourselves to an outline of some selected routines.

The main program CONVER is a control program and collects besides several,input statements and output routines - mainly the working routines

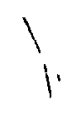

- SCRENF

- EIGENV

- CONTIN

- ICC

- PAPA

which are explained below.

The input consists of $5-7$ cards defining the nuclear charges and masses, the multipolarities, the transition energies and the subshells, for which internal conversion coefficients and/or particle parameters have to \&. 
be computed. They contain also the experimental electron-binding energies of the K-, L- and M-subshells. The output of the program (tape, cards or printer) can be regulated via input control indices. The subroutine SCRENF computes the atomic screening function using the Thomas-Fermi-Dirac model for the desired nuclear charge and stores it (Time: $14 \mathrm{sec}$ ). The computer times are given for the IBM 7094.

The subroutine EIGENV computes the eigen-value and the eigenfunctions of the bounded electron moving in the screened atomic potential (solution of the relativistic Dirac equation) and stores them. (Time: $8 \mathrm{sec}$ for K-shell, 14 sec for $M_{I}$-shell.)

The subroutine CONTIN computes the wave function of the ejected electron (moving in the screened atomic potential) and simultaneously computes the radial conversion matrix elements. The normalization and determination of the phase is performed also by this routine (Time: 8 - 20 sec for each partial wave, depending on the energy.)

Using these matrix elements, the internal conversion coefficients are determined by the subroutine ICC and the particle parameters by the subroutine PAPA (Time 0.1 sec).

Usually, the calculation of one conversion coefficient and/or particle parameter will not take more than $\frac{1}{2}-$ minute of an IBM 7094 computer and with this computer time it seems to be economical to compute each value directly, avoiding any interpolation procedure and error. The whole program is written in IBM FORTRAN-IV and uses only the most common library subroutines. The necessary computer memory storage is about 2700 words. Thus with the exception of the I/O routines, which can be modified easily, the program is compatible with all high-speed computers. 
The internal conversion coefficients and particle parameters of the $K$ and $L$ subshells have been recalculated for ten nuclei, i.e.

$$
Z=60,64,68,72,76,80,84,88,92 \text { and } 96
$$

and ten energies, i. e.

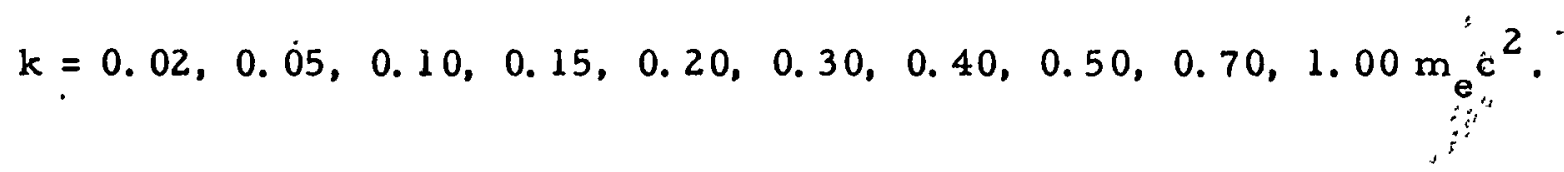

The four lowest orders of electric and magnetic multipole transitions have been considered.

The internal conversion coefficients and particle parameters of the M-subshells have been computed for seven nuclei, i.e.

$$
z=72,76,80,84,88,92 \text { and } 96
$$

and for ten energies (see above).

The screening of the atomic electrons has been taken into account on the basis of the Thomas-Fermi-Dirac model.

All static and dynamic effects of the finite nuclear size have been taken into account, assuming a sharp nuclear edge.

\section{Complete sets of Tables of Internal Conversion Coefficients and}

Particle Parameters containing the above mentioned data have been prepared and distributed among experimentalists who are interested in internal conversion phenomena.

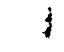


VIII. PERSONNEL OF PURDUE NUCLEAR PHYSICS LABORATORY

A. Faculty

R. M. Steffen, Professor of Physics

Principal Investigator

Z. W. Grabowski, Asst. Professor of Physics

S. M. Harris, Assoc. Professor of Physics

O. E. Johnson, Professor of Physics

R. P. Scharenberg, Assoc. Professor of Physics

P. C. Simms, Assoc. Professor of Physics

D. J. Tendam, Professor of Physics

B. Post-Doctoral Research Associates
G. F. Dell

B. T. Lucas

Z. Matumoto

H. C. Pauli

R. L. Rasera

J. W. Tippie

W. Zuckschwerdt
January 10, 1963

April 1, 1965

September 14, 1964

October 1, 1966

February 1, 1965

February 1, 1966

October 1, 1963
Terminated 
D. L. Coppage

S. W. Cosper

D. L. Deardorff

D. W. Grissmer .

J. W. Hemsky

J. C. Hill

J. L. Holm

T. Howell

W. C. King

H. A. Kuhlman

J. J. Lampka

H. M.'Nasir

D. R. Ober

D. E. Ohlms

- 'R. S. Raghavan

R. L. Rasera

G. E. Schilling

H. A. Smith

J. C. Spencer

B. B. Srivastava

C. L. Wiley

\section{Appointed}

February 1, 1964

June 1,1963

July 1, 1964

September 1, 1966

February 1, 1962

August 1, 1962

July 1,1967

Summer, 1967

June 20, 1966

July 1, 1966

September 1, 1966

July 1,1965

February 1, 1966

July 1, 1966

February 1, 1962

July 1, 1962

October 1,1967

July 1,1967

September 1, 1965

July 1,1964

January 1,1962

\section{Terminated}

present

July 31,1965

August 31,1967

present

August 31, 1966

January 3., 1966

present

presient

presents

present

June 30,1967

July 1,1967

February 29, 1968

present

November 7, 1964

February 1, 1965

January 31,1968

present

May 31,1966

August 31,1967

present

\section{Cyclotron Staff}

F. G. Hobaugh, Cyclotron Operator January 1, 1962

present

K. R. Runck, Night Operator , January 1,1962 July 1, 1967 
E. Electronics Shop

R. A. Dunham, Electronics Technician

February 1, 1965

present

2 - 3 part time undergraduate technicians

F. Plate Readers

R. R. Moore

January 1,1962

1 - 2 part time plate reader $s$

G. Secretarial

Mrs. B. Hunter, Secretary

Mrs. K. Schatz, Secretary ,

P. Fogarty, Secretary

Linda L. Stapleton

Patricia A. Sipple

. Carol S. Sne then
July 1,1962

July 1,1967

Mảyl, 1967

December 5, 1966

June 19, 1967

March 30, 1964

November 4, 1967

April 27, 1967

present

July 1,1967

June 16,1967

August 13,1967

present
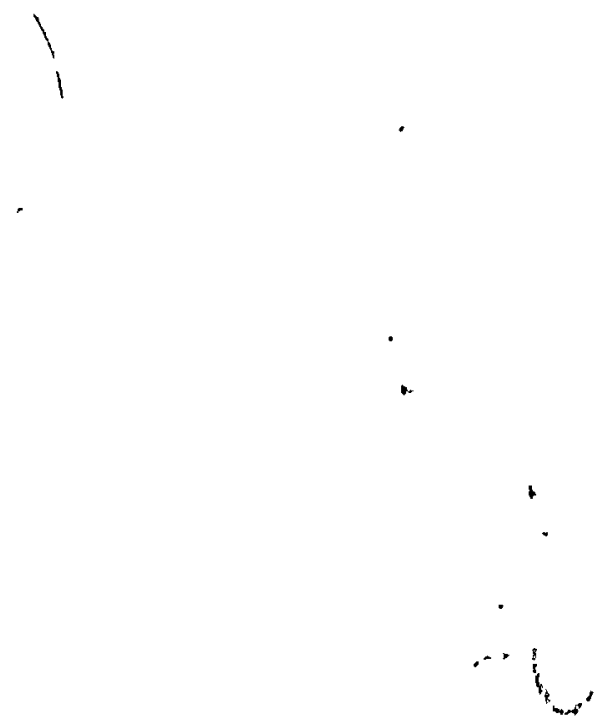
IX. ADVANCED DEGREES GRANTED

Academic Years $1964-1967$

S. W. Cosper, Ph. D.

D. L. Deardorff, Ph. D.

J. W. Hemsky, Ph. D.

J. C. Hill, Ph. D.

H. M. Nasir, Ph. D.

D. R. Ober, Ph. D.

R. S. Raghavan, Ph. D.

R. L. Rasera, Ph. D.

B. B. Srivastava, Ph. D.
August, 1965

January, 1968

January, 1967

June, 1966

August, 1967

January, 1968

January, 1965

January, 1965

June, 1967

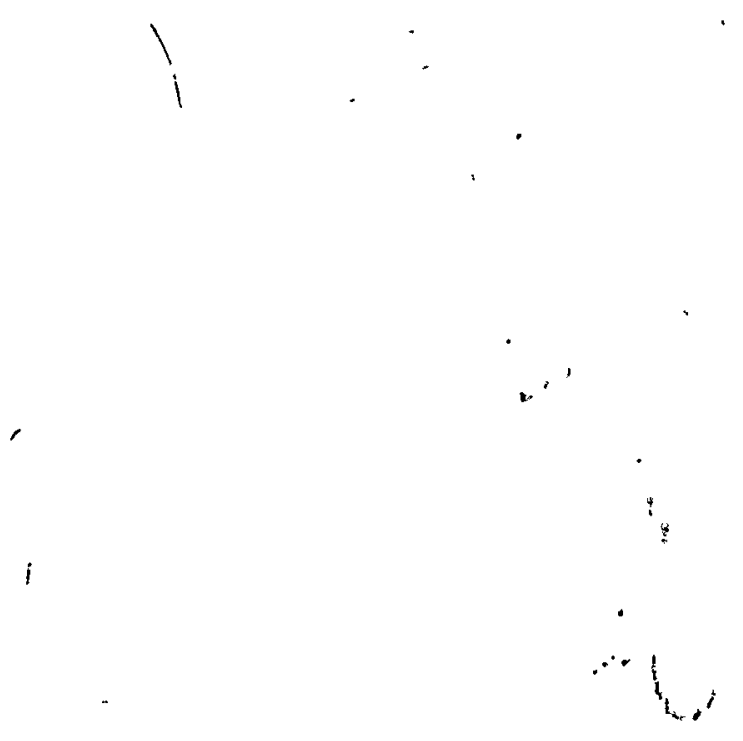




\begin{abstract}
x. LIST OF PUBLICATIONS
(not including abstracts of papers presented at meetings)

October 1,1964 to September 30,1967

A. Papers Published in Professional Journals
\end{abstract}

1. Large-Spin Rotational States of Deformed Nuclei. S. M. Harris. Phys. Rev. Letters 13, 663 (1964).

2. The $\mathrm{F}^{19}(\mathrm{~d}, \alpha) \mathrm{O}^{17}$ Reaction at $9.2 \mathrm{MeV}$. S. W. Cosper, B. T. Lucas, and O. E. Johnson. Phys. Rev. 138, B51 (1965).

3. Higher-Order Corrections to the Cranking Model. S. M. Harris. Phys. Rev. 138, B509 (1965).

4. An Experimental Study of the $(2 I+1)$ Rule Using the $(d, \alpha)$ Reaction on $\mathrm{F}^{19}, \mathrm{Na}^{23}, \mathrm{Al}^{27}$, and $\mathrm{P}^{31}$. S. W. Cosper and O. E. Johnson. Phys. Rev. 138, B610 (1965).

5. Beta-Decay and Nuclear Structure. R. M. Steffen. Paper: Proceedings of the Nuclear Physics Symposium, Calcutta (India), February (1965).

6. A Study of Second Excited $2^{+}$States of Some Even-Even Nuclei by BetaGamma Angular Correlations. R. S. Raghavan, Z. W. Grabowski, and R. M. Steffen. Phys. Rev. 139, B 1 (1965).

7. Beta-Gamma Circular Polarization Correlation of $\mathrm{Sb}^{122}$. Z. W. Grabowski, 'R. S. Raghavan, and R. M. Steffen. Nuclear Physics $\underline{70}$, 170 (1965).

8. Search for Higher-Order Effects in Allowed B-Transitions. Z. W. . - Grabowski, R. S. Raghavan, and R. M. Steffen. Phys. Rev. $\frac{139,}{i}$, B24
(1965).

9. The Nuclear Matrix Elements in the First-Forbidden 2. $2 \mathrm{MeV} B-$ Transition of La ${ }^{140}$. R. M. Singru, P. C. Simms, and R. M. Steffen. |Phys. Rev. 141, 1078 (1966). 
10. Some $(d, \alpha)$ Differential Cross Sections for $\mathrm{Na}^{23}, \mathrm{Al}^{27}$, and $\mathrm{P}^{31}$ at about $9.3 \mathrm{MeV}$. S. W. Cosper, B. T. Lucas, and O. E. Johnson. Phys. Rev. 139, B763 (1965).

11. Transverse Polarization of K-Conversion Electrons following the $\beta$ Decay of $\mathrm{Au}^{198}$. R. L. Rasera and R. M. Steffen. Phys. Rev. 140 B 1177 (1965).

12. Decay of Eu ${ }^{145}$. John C. Hill. Phys. Rev. 153, 1312 (1967).

13. Optical-Model and Distorted-Wave Analyses of Some 18-MeV AlphaParticle Scattering Cross Sections for $A^{40}, s^{32}, P^{31}, \mathrm{Na}^{23}$, and $\mathrm{Ne}^{20}$. B. T. Lucas, S. W. Cosper, and O. E. Johnson. Phys. Rev. 144, 972 (1966).

14. Higher-Order Effects in $\beta-\gamma$ Correlations Involving Allowed $\beta-$ Transitions, S. Cipolla, H. Nasir, Z. W. Grabowski, and R. M. Steffen. Phys. Rev. 146, 877 (1966)

15. Elastic and Inelastic Scattering of $9.8 \mathrm{MeV}$ Deuterons from $\mathrm{P}^{31}$. B. T. Lucas, and O. E. Johnson. Phys. Rev. 145, 887 (1966).

16. Study of the $P^{31}\left(\alpha, d_{0,1}\right) S^{33}$ Reactions at 18. $7 \mathrm{MeV}$. B. B. Srivastava, S. W. Cosper, and O. E. Johnson. Phys. Rev. 153, 1221 (1967).

17. Measurement and DWBA Analyses of the 18.7 MeV Cross Sections for the $\mathrm{Na}^{23}\left(\alpha, \mathrm{d}_{0,1}\right) \mathrm{Mg}^{25}$ Reactions. B. B. Srivastava and O. E. Johnson. Phys. Rev. 156, 1219 (1967).

18. Measurement and DWBA Analyses of the 18.7-MeV Cross Sections for the $A I^{27}\left(\alpha, d_{0,1}\right) S^{29}$ Reactions. B. B. Srivastava and O. E. Johnson. Phys. Rev. in press.

19. Quantum Mechanical Model of Mossbauer Line Narrowing. Samuel M. Harris. Phys. Rev. 163, 280 (1967).

20. Internal Conversion Particle Parameters in Deformed Rare Earth Nuclei. R. M. Steffen and Z. W. Grabowski. Proceedings of International Conference on Nuclear Structure, Tokyo (Japan), September $7-13,1967$. 
21. Conversion Electron Particle Parameters in the Decay of $\mathrm{Eu}^{152}$ and $E u^{154}$. H. M. Nasir, Z. W. Grabowski, and R. M. Steffen. Phys. Rev. 162, 1118 (1967).

22. The Magnetic Hyperfine Field at Hf Nuclei in an Iron Lattice. A. J. Becker and R. M. Steffen. Proceedings of the International Conference on Hyperfine Interactions Detected by Nuclear Radiations, Asilomar (Calif.) August 25 - 30, 1967. (Nor th Holland Publishing Co.)

23. Static Quadrupole Moment of the $2^{+}$State in ${ }^{114} \mathrm{Cd}$ Determined by Coulomb Excitation. G. Schilling and R. P. Scharenberg, and J. W. Tippie. Phys. Rev. Letters 19, 318 (1967).

24. Measurements and Distorted-Wave Born-Approximation Analyses of Some 13. 9-MeV Differential Cross Sections for the $\mathrm{N}^{14}\left(\mathrm{He}^{3}, \alpha\right) \mathrm{N}^{13}$ Reactions. B. T. Lucas, D. R. Ober, and O. E. Johnson. Phys. Rev, in press.

25. Isospin Conservation in the ${ }^{40} \mathrm{Ca}(\mathrm{d}, \alpha)^{38} \mathrm{~K}$ Reaction. J. W. Hemsky, 'E. Bleuler, and D. J. Tendam. Nuclear Physics A105, 665 (1967) A

26. Study of Some $\mathrm{C}^{12}\left(\mathrm{He}^{3}, \alpha\right) \mathrm{C}^{11}$-Reactions at $13.9 \mathrm{MeV}$. D. R. Ober and O. E. Johnson. Phys. Rev. in press. 


\section{B. Reports and Tables}

1. The Calibration of the Purdue Broad Range Magnetic Spectrograph and the Incident-Beam Energy Analyzing Magnet.

E. Bleuler, D. J. Tendam, J. W. Hemsky and C. L. Wiley. April, 1966.

2. Angular Correlation Handbook.

Vol. I. Unperturbed Directional Correlations

R. M. Steffen. August, 1965.

3. Lectures on Angular Correlations of Nuclear Radiations .

R. M. Steffen, Tata Institute of Fundamental Research, Bombay, 1965.

4. Tables of Internal Conversion Coefficients and Particle Parameters.

H. C. Pauli.

5. Research in Nuclear Physics, Progress Report No. 15, June 15, 1965

6. Research in Nuclear Physics, Progress Report No. 16, June 15, 1966

7. Research in Nuclear Physics, Progress Report No. 17, June 15, 1967 